\title{
Source-independent efficient wavefield inversion
}

\author{
Chao Song and Tariq Alkhalifah \\ Department of Physical Science and Engineering, \\ King Abdullah University of Science and Technology, \\ Thuwal 23955-6900, Saudi Arabia, \\ *E-mail: chao.song@kaust.edu.sa
}

4 May 2020

\section{SUMMARY}

Full-waveform inversion (FWI) is an effective tool to retrieve a high-resolution subsurface velocity model. The source wavelet accuracy plays an important role in reaching that goal. So we often need to estimate the source function before or within the inversion process. Source estimation requires additional computational cost, and an inaccurate source estimation can hamper the convergence of FWI. We develop a sourceindependent waveform inversion utilizing a recently introduced wavefield reconstruction based method we refer to as efficient wavefield inversion (EWI). In EWI, we essentially reconstruct the wavefield by fitting it to the observed data as well as a wave equation based on iterative Born scattering. However, a wrong source wavelet will induce errors in the reconstructed wavefield, which may lead to a divergence of this optimization problem. We use a convolution-based source-independent misfit function to replace the conventional data fitting term in EWI to formulate a source-independent EWI (SIEWI) objective function. By convolving the observed data with a reference trace from the predicted data and convolving the predicted data with a reference trace from the observed data, the influence of the source wavelet on the optimization is mitigated. In SIEWI, this new formulation is able to mitigate the cycle-skipping issue and the source wavelet uncertainty, simultaneously. We demonstrate those features on the Overthrust model and a modified Marmousi model. Application on a 2D real dataset also shows the effectiveness of the proposed method.

Key words: Source-independent; Efficient wavefield inversion; Cycle-skipping; Inverse crime

\section{INTRODUCTION}

Full-waveform inversion (FWI) is a highly non-linear problem which aims at minimizing the misfit between predicted and observed data to retrieve a high-resolution velocity model (Tarantola 1984 Pratt et al. 1998, Virieux \& Operto 2009). The knowledge of the true source function is an important prerequisite to achieve a successful wave equation based inversion. Thus, the source wavelet estimation becomes an essential step for practical FWI applications. It is often, however, a difficult task when dealing with noisy data, imperfect acquisition, or unpreserved amplitude during the data preprocessing (Chi et al. 2018). To get a good estimation of the source wavelet in the field data, we often utilize the direct arrivals in streamer data to extract the source signature. However, in shallow water, ocean bottom cable survey or land exploration, the direct arrivals are usually contaminated with reflections and near surface noise (Skopintseva et al. 2016), and the source wavelet extraction is not reliable. We can also estimate the source signature during the model update process (Pratt 1999), and this approach is widely used in the FWI applications (Kamei et al. 2013, 2014). However, a simultaneous inversion of the velocity and the source wavelet may diverge when the initial velocity is poor and low frequency components in the data are missing.

To mitigate the the source estimation error influence, deconvolution-based source-independent objective functions were developed (Lee \& Kim 2003 Zhou \& Greenhalgh 2003 Choi et al. 2005. Xu et al. 2006). For the crosshole seismic data, they mitigate the source wavelet effect by normalizing the data amplitude with a reference wavefield. However, the reference trace needs to be chosen wisely. If we implement FWI in the time-domain, it requires an additional Fourier transform. A 


\section{Song and Alkhalifah}

convolution-based approach also requires to choose a reference trace from the observed and predicted data Choi \& Alkhalifah 2011). In their implementation, they convolve the observed data with the selected predicted data reference trace, and vice versa, they convolve the predicted data with the selected observed reference trace to formulate the objective function. In this source-independent objective function, the source function of both observed and predicted data are equally distributed in both terms, and thus, the effect of the source wavelet is mitigated. This approach is also successfully applied in the passive seismic inversion, in which the source function is unknown (Wang \& Alkhalifah 2018). As the source origin time is unknown in passive seismic scenario, this source-independent objective function can be used to update the velocity model. Besides the deconvolution- and convolution-based methods, a new source-independent FWI using an amplitude-semblance objective function was proposed. In this approach, no reference trace selection is required (Chi et al. 2018). All of these approaches were focused on optimization problems with respect to the velocity model, often referred to as FWI.

Besides the uncertainty of the source wavelet, there is always a greater challenge from cycle-skipping issue in FWI, a direct result of the high non-linearity of the conventional $l_{2}$ norm objective function with respect to the velocity model. Many methods developed recently aim to solve the cycle-skipping problem and retrieve the low-wavenumber model update (Shin \& Cha 2008, Ma \& Hale 2013, Wu et al. 2014 |Biondi \& Almomin 2014, Warner \& Guasch 2016, Alkhalifah \& Wu 2016). Among these methods, wavefield reconstruction inversion (WRI) was proposed to reduce the cycle-skipping issue by relaxing the wave equation constraint transforming it to a regularization term (Leeuwen \& Herrmann 2013, 2015). To mitigate the source wavelet error influence in WRI, a source estimation method is specially designed for WRI (Fang et al. 2018). In WRI, the wavefield is still highly dependent on the velocity, and this dependency can be mitigated by replacing the velocity with a modified source function, which includes the veloctiy perturbation, we refer to as efficient wavefield inversion (EWI) (Alkhalifah \& Song 2019). EWI is able to calculate the medium parameter perturbations directly, and it also proves its effectiveness in complex media (Song et al. 2019a Song \& Alkhalifah 2020a).

In this paper, we propose to combine the convolution-based source-independent approach and EWI to remove the source function effect in inverting the wavefield. This source-independent EWI (SIEWI) formulation replaces the data fitting term by a convolution-based source-independent misfit function represented by multiplications in the frequency domain. This new formulation not only maintains the advantage of efficiency and cycle-skipping immune feature in EWI, but also mitigates the source wavelet effect. We test the proposed approach on the Overthrust and a modified Marmousi model, and the results demonstrate the accuracy of the proposed method. An application on a $2 \mathrm{D}$ real dataset also demonstrates the effectiveness of SIEWI.

\section{THEORY}

\subsection{Full-waveform inversion (FWI)}

Conventional FWI measures the misfit between observed and predicted data using the wave equation as a constraint on the wavefield, as follows (Tarantola 1984):

$$
J(V)=\min \frac{1}{2} \sum_{i} \sum_{j}\left\|d_{i, j}-C u_{i}\right\|_{2}^{2} \quad \text { s.t. } \quad L(V) u_{i}=f_{i}
$$

where $i$ and $j$ denote the source and receiver indexes in which we sum over. $C$ acts as the mapping operator projecting the background wavefield $u_{i}$ vector to the receiver positions. $L(V)=\left(V_{0}+V\right) \omega^{2}+\nabla^{2}=L_{0}+\omega^{2} V$ is the modelling operator, and $L_{0}=V_{0} \omega^{2}+\nabla^{2}$ corresponds to the background modelling operator. $V$ denotes the squared slowness perturbation, and we set it to zero at the beginning of the inversion process. According to the adjoint-state method, the squared slowness gradient is evaluated by (R.E.Plessix 2006):

$$
\nabla_{V} J=-\omega^{2} \sum_{i} \sum_{j} \Re\left\{u_{i}\left(L^{-1}\right)^{T}\left(C^{T} \Delta d_{i, j}^{*}\right)\right\}
$$

where $\Delta d_{i, j}$ represents the data residual, and $\left(L^{-1}\right)^{T}\left(C^{T} \Delta d_{i, j}^{*}\right)$ represents the back-propagated wavefield vector. $T$ denotes ordinary matrix transpose, the superscript $*$ represents the complex conjugate, and $\Re$ means taking the real part of a complex value. Equation 2 shows that the gradient of FWI is obtained by calculating the product of the back-propagated wavefield and background wavefield (Choi et al. 2008). FWI requires a large amount of computational cost and disk memory in storing the background wavefields. The backgournd modelling operator used to calculate the background wavefield is dependent on the velocity. This dependency leads to the high non-linearity of the $l_{2}$ norm objective function. 


\subsection{Wavefield reconstruction inversion (WRI)}

WRI mitigates these issues by transforming the wave equation to an additional regularization term (Leeuwen \& Herrmann 2013), as follows:

$$
E\left(V, u_{i}\right)=\min \frac{1}{2} \sum_{i} \sum_{j}\left\|d_{i, j}-C u_{i}\right\|_{2}^{2}+\frac{\epsilon^{2}}{2} \sum_{i}\left\|L(V) u_{i}-f_{i}\right\|_{2}^{2} .
$$

By controlling the positive weighting factor $\epsilon^{2}$, the accuracy of the wave equation is relaxed to enforce more data fitting even with an inaccurate velocity model. However, in this formulation, the wavefield still has the dependency on the modelling operator $L(V)$. To mitigate this dependency, we linearise the objective function by replacing the true source function with a modified one (Alkhalifah \& Song 2019).

\subsection{Efficient wavefield inversion (EWI)}

EWI presents an optimization for the wavefield independent of the velocity perturbation in the wave equation operator, given by :

$$
E\left(f_{e i}, u_{i}\right)=\min \frac{1}{2} \sum_{i} \sum_{j}\left\|d_{i, j}-C u_{i}\right\|_{2}^{2}+\frac{\epsilon^{2}}{2}\left\|L_{0} u_{i}-f_{e i}\right\|_{2}^{2},
$$

where, $f_{e i}$ represents the modified source function. In equation 4 , there are two independent variables: the wavefield $u_{i}$ and the modified source function $f_{e i}$. We calculate the wavefield using a linear equation given by an augmented wave equation:

$$
\left(\begin{array}{c}
\epsilon L_{0} \\
C
\end{array}\right) u_{i}=\left(\begin{array}{c}
\epsilon f_{e i} \\
d_{i, j}
\end{array}\right) .
$$

The least-squares solution of equation 5 is to solve $\left(C^{T} C+\epsilon^{2} L_{0}^{*} L_{0}\right) u_{i}=C^{T} d_{i, j}+\epsilon^{2} L_{0}^{*} f_{e i}$. $L_{0}^{*}$ means the conjugate transpose form of $L_{0}$. In each frequency, the modelling operator $L_{0}$ in equation 5 remains stationary. If the wavefield $u$ is accurately reconstructed, the modified source $f_{e}$ can be derived using:

$$
L u_{i}=f_{i} \rightarrow\left(L_{0}+\omega^{2} V\right) u_{i}=f_{i} \rightarrow L_{0} u_{i}=f_{e i}=f_{i}-\omega^{2} V u_{i} .
$$

As the objective function $E$ is a quadratic function of $f_{e i}$ in equation 4 , by setting $\frac{\partial E}{\partial f_{e i}}=0, f_{e i}$ can be evaluated using:

$$
f_{e i}=L_{0} u_{i} \text {. }
$$

Initially, the wavefield only has single scattering components from equation 5 . As medium parameter perturbations are stored in $f_{e}$, inner iterations between equations 5 and 7 are used to add the multiscattering components to the wavefield corresponding to secondary sources. This is discussed in details in Alkhalifah \& Song (2019). The squared slowness perturbation is not treated as an unknown parameter in EWI. After optimizing the two independent variables $u$ and $f_{e}$ using inner iterations, $V$ can be directly calculated using a direct division process according to equation 6

$$
V=\frac{\left(f_{i}-f_{e i}\right)}{\omega^{2} u_{i}}
$$

The squared slowness perturbation $V$ can be calculated by each individual source. However, a single source has a limited illumination to the subsurface, so we calculate $V$ by stacking all the sources, given by:

$$
V=\sum_{i} \frac{\left(f_{i}-f_{e i}\right)}{\omega^{2} u_{i}} \approx \sum_{i} \frac{\left(f_{i}-f_{e i}\right) u_{i}^{*}}{\omega^{2} u_{i} u_{i}^{*}+\lambda}
$$

where $\lambda$ is a small positive real number to avoid dividing by zero. Usually, we use one percentage of the maximum value of the wavefield amplitude $u_{i}^{*} u_{i}$. Multiscattering information provides more illumination to the model, so $V$ benefits from more low wavenumber updates (Alkhalifah \& Wu 2016; Song et al. 2019b). However, the calculated squared slowness perturbation is only accurate when the wavefield reconstruction result from equation 5 is reliable.

\subsection{Source-independent efficient wavefield inversion (SIEWI)}

The accuracy of the wavefield reconstruction relies on the initial velocity and the source function. If a wrong source wavelet is used in equation 5, the reconstructed wavefield will have a significant error compared to the true wavefield. In this case, the error in $u$ will propagate to $f_{e}$ and $V$ through equations 5 to 8 , and hamper the convergence of the inversion process. This is a great challenge in real data applications, because the true wavelet is difficult to retrieve. To solve this problem, we propose to use a source-independent EWI (SIEWI). In time-domain, a source-independent based objective function is stated as (Choi \& Alkhalifah 2011):

$$
J=\min \frac{1}{2} \sum_{i} \sum_{j}\left\|\widehat{d}_{i, j} \circledast d_{i, k}-d_{i, j} \circledast \widehat{d}_{i, k}\right\|_{2}^{2} .
$$




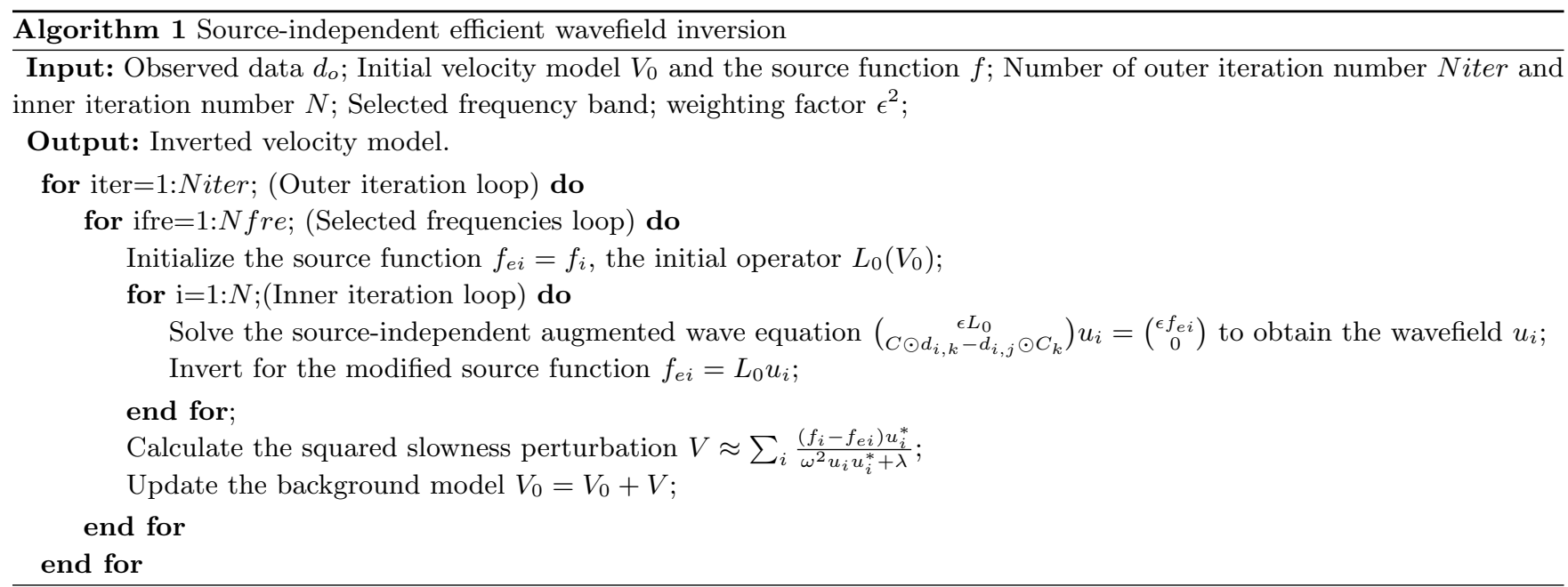

$\widehat{d}_{i, j}$ and $d_{i, j}$ represent the predicted and the observed data, and $\widehat{d}_{i, k}$ and $d_{i, k}$ are defined as the reference trace at the $k$ th receiver position from the predicted and observed data, respectively. The symbol $\circledast$ represents the convolution operation in time. For each shot gather, the predicted data are convolved with a selected observed data reference trace, and vice versa. By doing so, the source function is represented equally in both terms, and its effect is removed from the objective function (Choi \& Alkhalifah 2011). However, with this objective function we may still encounter the cycle-skipping problem caused by a poor initial velocity. We formulate an SIEWI objective function by replacing the $l_{2}$ norm data fitting term with a sourceindependent objective function. This new formulation aims to avoid the source estimation and mitigate the cycle-skipping problem, simultaneously. It can be formulated in the frequency domain using the same reference trace concept as:

$$
E\left(f_{e i}, u_{i}\right)=\min \frac{1}{2} \sum_{i} \sum_{j}\left\|C u_{i} \odot d_{i, k}-d_{i, j} \odot C_{k} u_{i}\right\|_{2}^{2}+\frac{\epsilon^{2}}{2} \sum_{i}\left\|L_{0} u_{i}-f_{e i}\right\|_{2}^{2} .
$$

In equation 11. $C u_{i}$ is equivalent to $\widehat{d}_{i, j}$, which denotes the predicted data. $C_{k}$ is a mapping operator which just takes the value from the wavefield $u_{i}$ at the reference trace location, $k$. So we have $\widehat{d}_{i, k}=C_{k} u_{i}$. In frequency domain, the convolution process becomes an element by element multiplication, and we use the symbol $\odot$ to denote this. The wavefield $u$ and modified source $f_{e}$ are still two independent variables in SIEWI. As a result, we can calculate the wavefield by solving a source-independent augmented wave equation:

$$
\left(\begin{array}{c}
\epsilon L_{0} \\
C \odot d_{i, k}-d_{i, j} \odot C_{k}
\end{array}\right) u_{i}=\left(\begin{array}{c}
\epsilon f_{e i} \\
0
\end{array}\right) .
$$

We solve $\left(P_{k}^{*} P_{k}+\epsilon^{2} L_{0}^{*} L_{0}\right) u_{i}=\epsilon^{2} L_{0}^{*} f_{e i}$ to get the least-squares solution of equation 11 Here, $P_{k}=C \odot d_{i, k}-d_{i, j} \odot C_{k}$ This source-independent augmented wave equation is able to reconstruct the wavefield with limited source function influence. Then we can calculate the modified source function and squared slowness perturbation using equations 7 and 9 , In equation 9 , we calculate the squared slowness perturbation $V$ pixel by pixel, while the source function $f_{i}$ is zero everywhere except at the source location in the space domain. As a result, the accuracy of $f_{i}$ barely affects the calculation of $V$ as long as the wavefield $u_{i}$ can be accurately reconstructed by SIEWI. Finally, the background model is updated:

$$
V_{0}=V_{0}+V \text {. }
$$

\section{IMPLEMENTATION}

In this section, we explain the concept and functionality of the inner and outer iterations used on EWI and elaborate on the algorithm within the context of the source independent implementation.

\subsection{The inner iterations}

The objective function of either EWI or the SIEWI (in equations 4 and 10 has two terms. The first one aims to extract the wavefield information from the data. The second one is devoted to making sure this wavefield fits the wave equation corresponding to the current model. To extract an accurate wavefield that fits the data, and yet satisfies the wave equation for an inaccurate background velocity, the EWI objective function uses a modified source function $f_{e}$. Specifically, $f_{e}$ holds the the velocity perturbations information necessary to produce a wavefield that fits the data for an inaccurate background velocity 
(a)

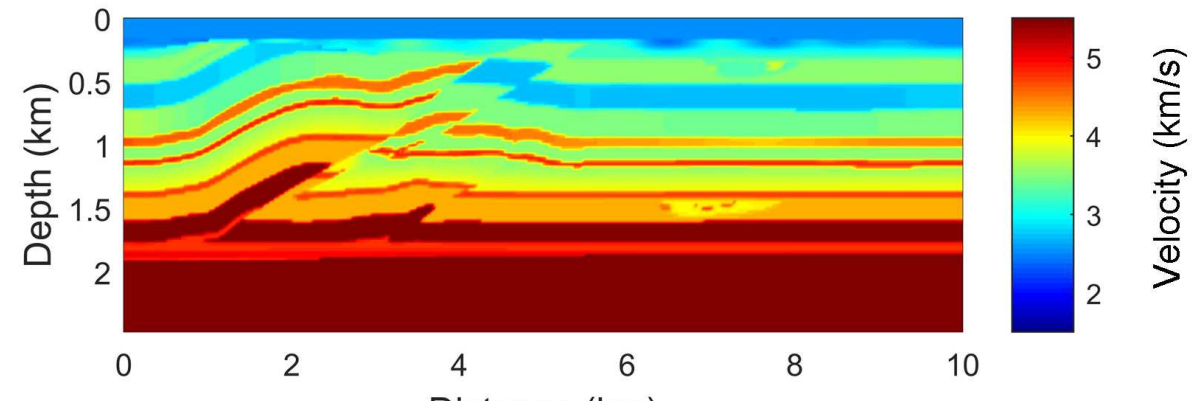

(b)

Distance $(\mathrm{km})$

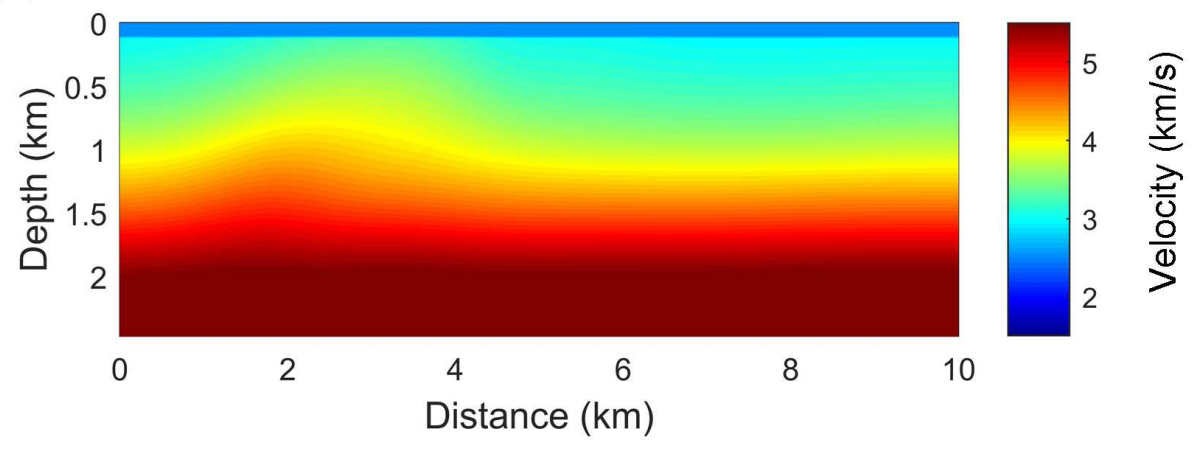

Figure 1. The true (a), and initial (b) Overthrust models.

(a)

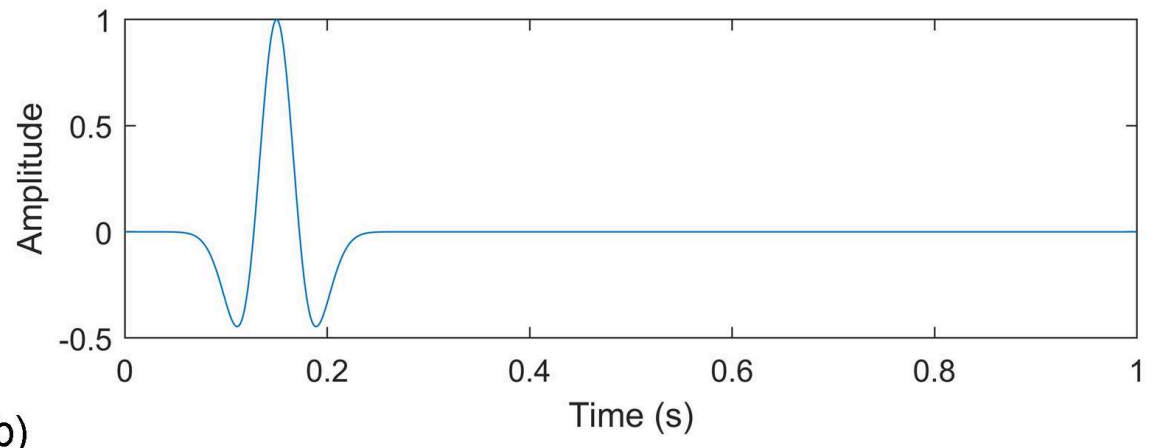

(b)

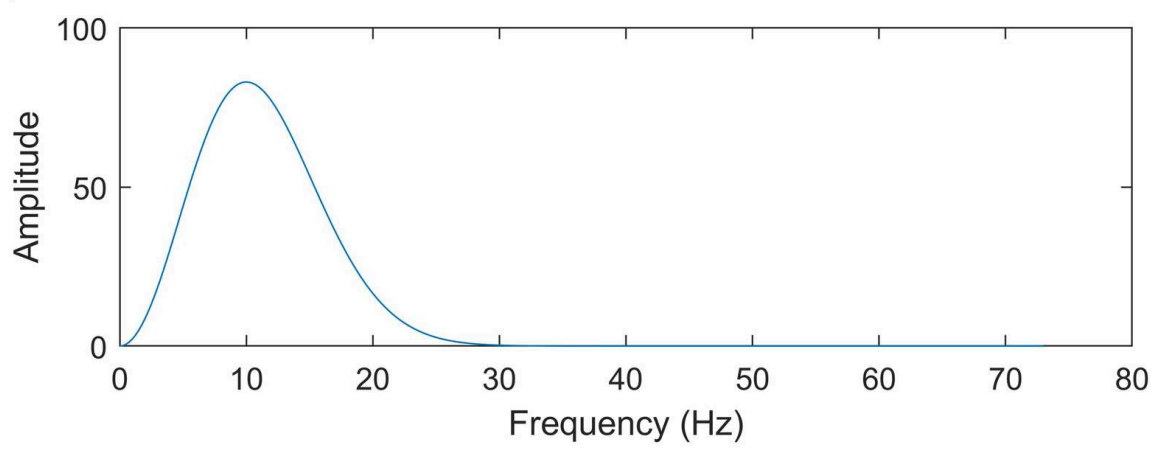

Figure 2. The true wavelet (a), and its corresponding frequency spectrum (b). 
(a)
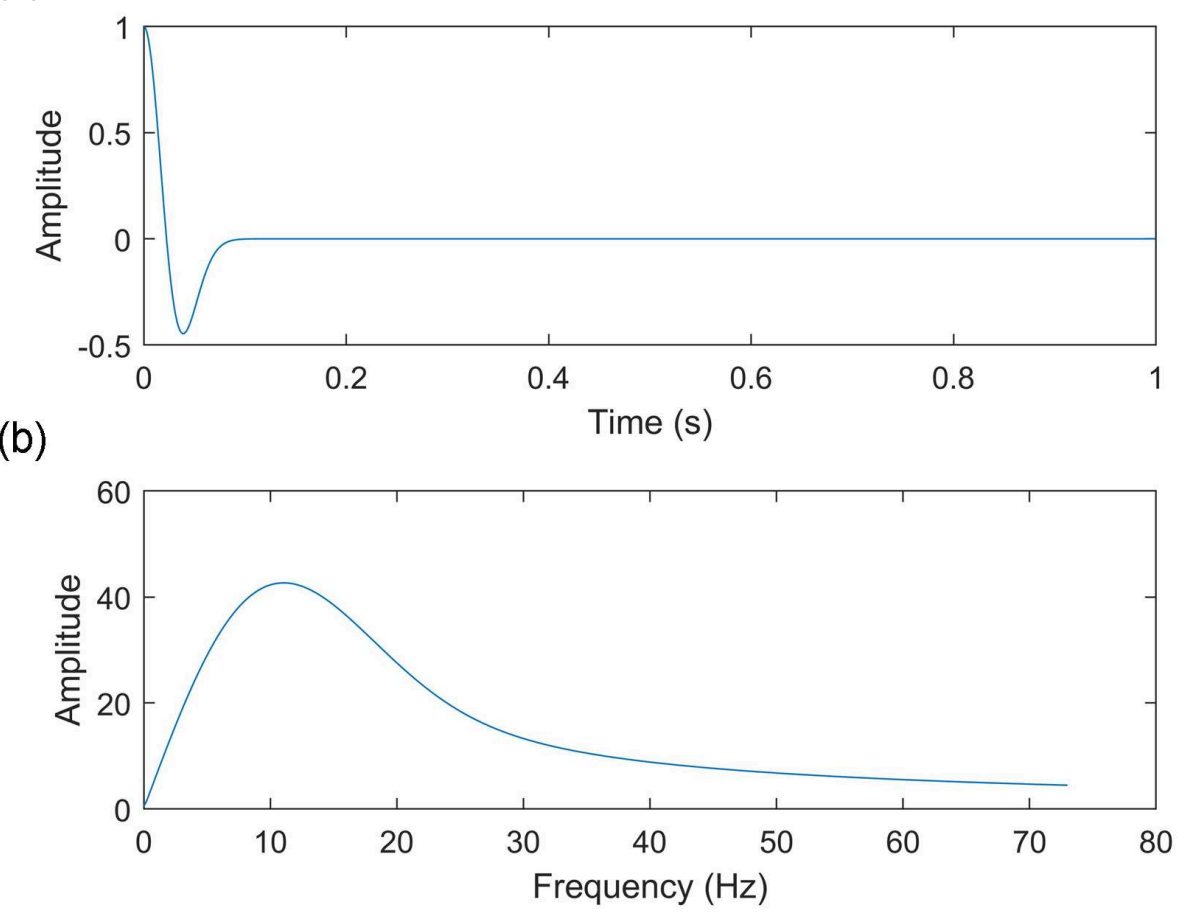

Figure 3. The wrong wavelet (a), and its corresponding frequency spectrum (b).

model. In the first inner iteration, we start with $f_{e}=f$ (the true source). In this case, the calculated wavefield $u$ is a compromise between fitting the wave equation and data by controlling $\epsilon^{2}$. For a smooth background model, the resulting wavefield and modified source function reflect energy corresponding to single scattering of what is required to fit the data. If only a single inner iteration is used, the original EWI reduces to WRI in the wavefield inversion part of the implementation, with respect to inversion for the source. Within each selected frequency, any update in the velocity requires a new matrix inversion. EWI introduces an inner iteration to update $u$ and $f_{e}$, and this operation is equivalent to adding scattering components to the wavefield. As $f_{e}$ is updated with the perturbations of the model, these perturbations act as secondary sources in $f_{e}$ weighted by $\epsilon^{2}$. The larger the $\epsilon^{2}$, the more secondary scattering we add to the wavefield. However, the larger the $\epsilon^{2}$, the smaller the

(a)

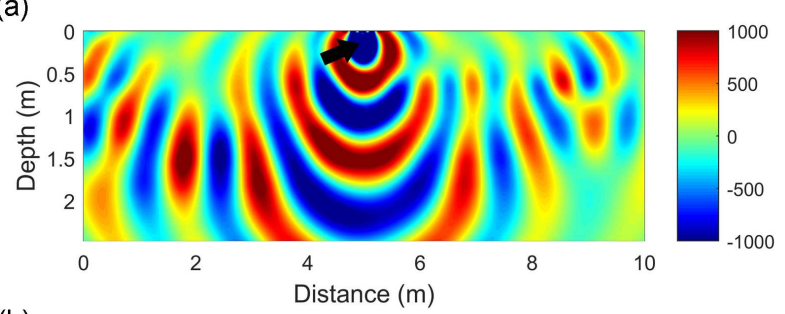

(b)

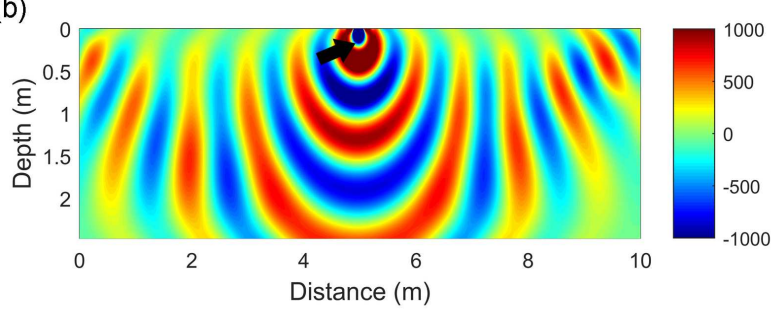

(c)

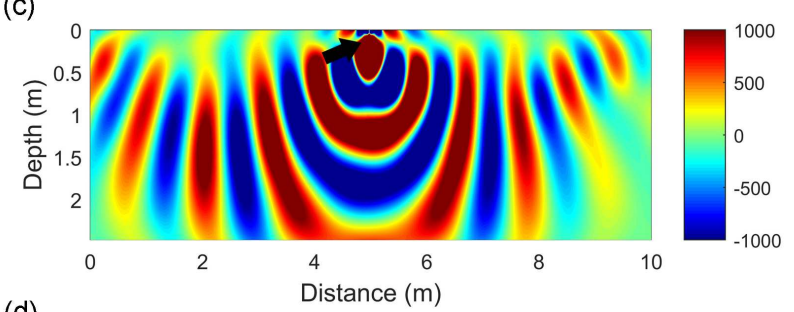

(d)

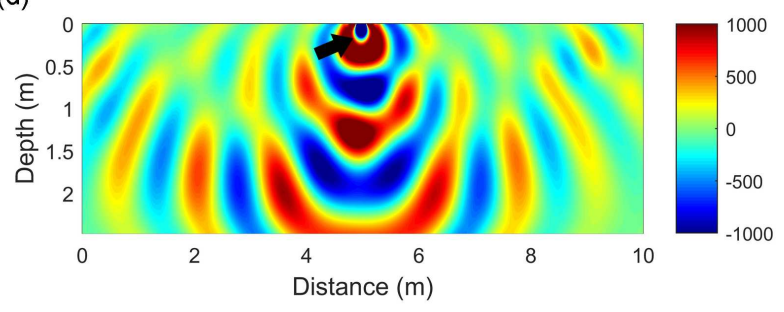

Figure 4. Wavefields constructed using the wave equation for the true (a), and initial (b) velocity models. Wavefields constructed using the original augmented wave equation (c), and the source-independent augmented wave equation (d), both with the inaccurate source. 
(a)
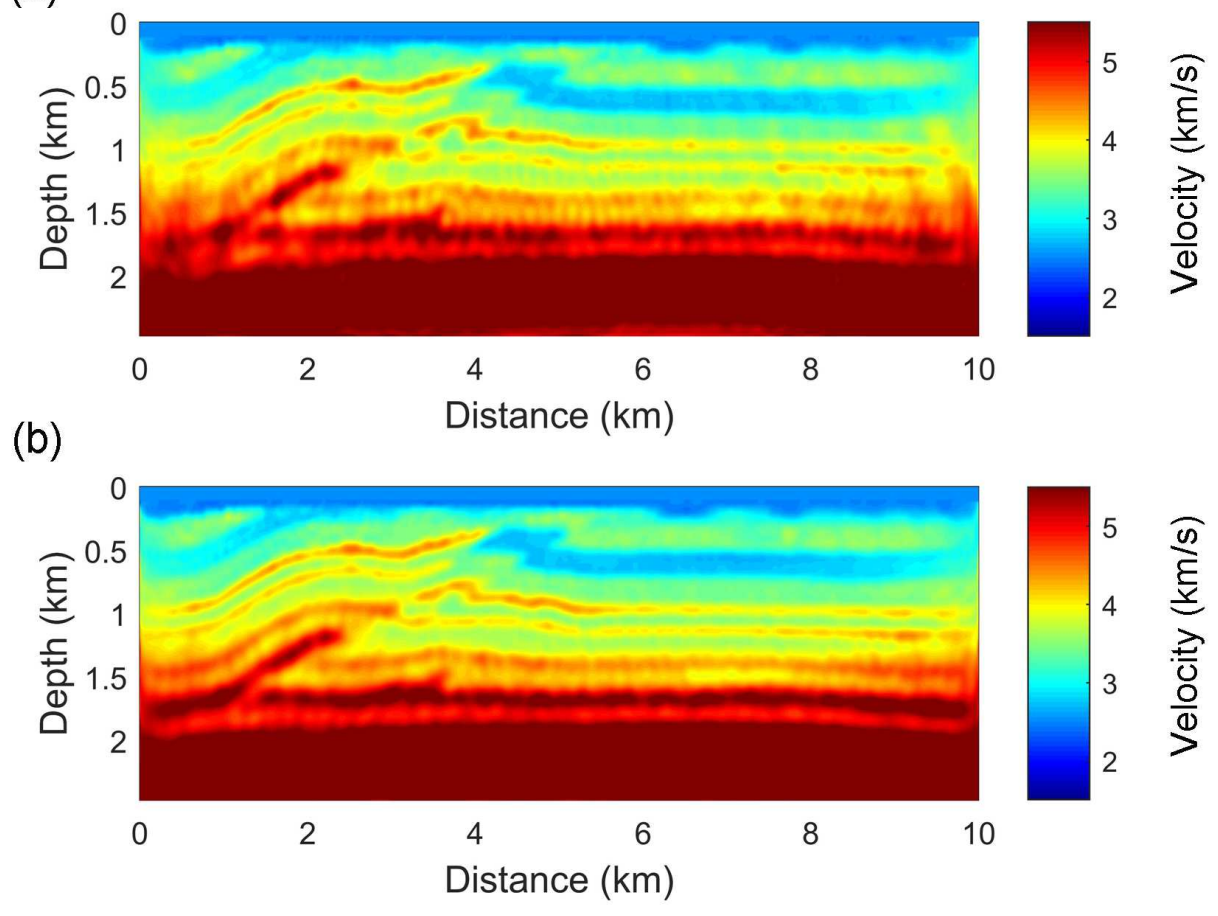

Figure 5. The inverted velocity using WRI (a), and FWI (b) after one sweep over the all frequencies with one iteration per frequency using the true wavelet.

perturbations we extract from fitting the data. How much of the scattering we extract from the data to $f_{e}$ and how much of these secondary sources are used to develop the wavefield need to be considerably balanced. The wavefield initially will contain only single scattering components between the receivers and secondary sources in $f_{e}$. Another iteration of the linear inversion for $u$, with the updated $f_{e}$ will add second-order scattering energy to the wavefield (Alkhalifah 2019). In practice, two or three inner iterations is enough, as scattering energy higher than the second-order is reasonably weak to effectively contribute the velocity update. Thus, in all the examples shown in this paper, we use two inner iterations.

\subsection{The outer iterations}

Although inner iterations in EWI can include multi-scattered energy in the reconstruted wavefield $u$, EWI is not completely immune to the cycle-skipping problem when the velocity perturbation is very large. In this case, we use outer iterations to update the velocity model throughout the whole selected frequency band to gradually improve the inversion result (Song \&

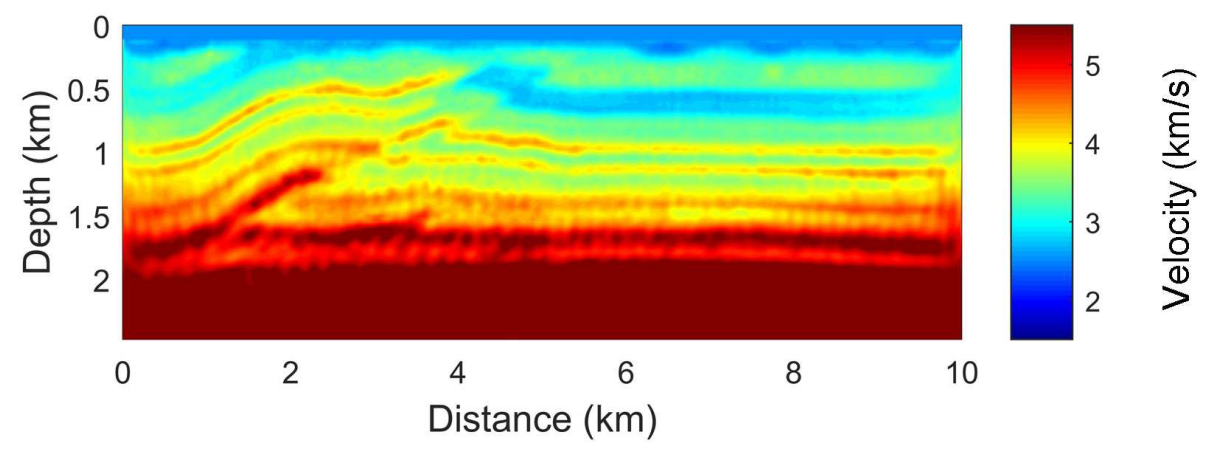

Figure 6. The inverted velocity using EWI after one sweep over the all frequencies with one iteration per frequency with the true wavelet. 
(a)

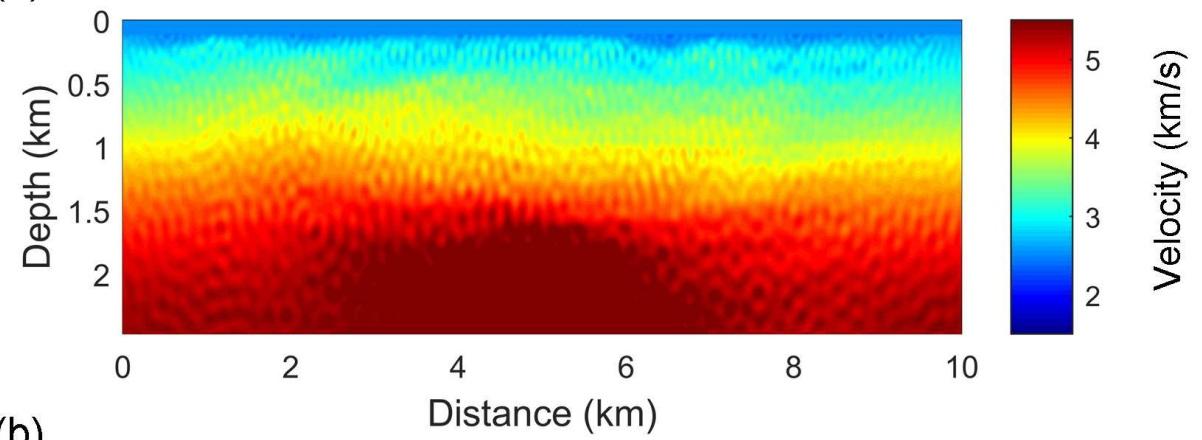

(b)

Distance $(\mathrm{km})$

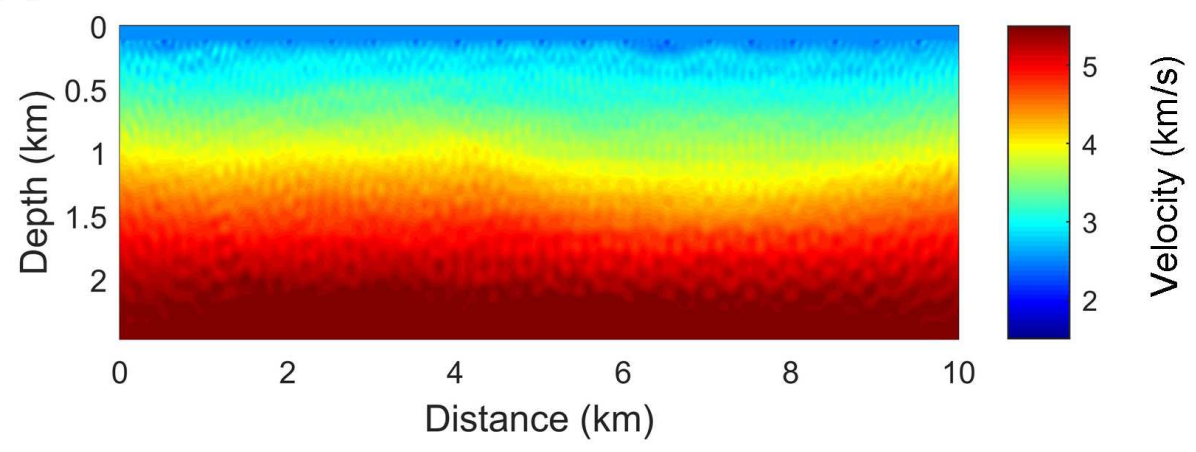

Figure 7. The inverted velocity using WRI (a), and FWI (b) after one sweep over the all frequencies with one iteration per frequency with the wrong wavelet.

(a)

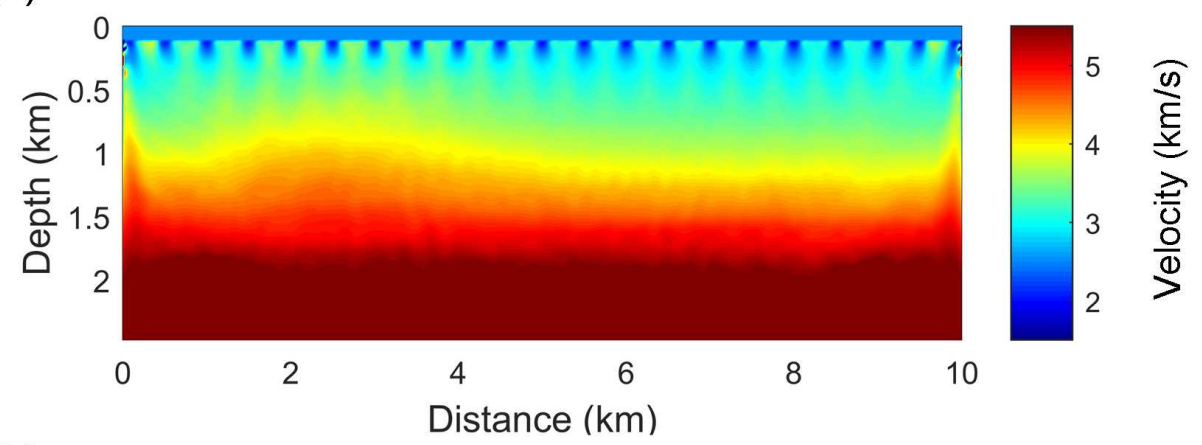

(b)

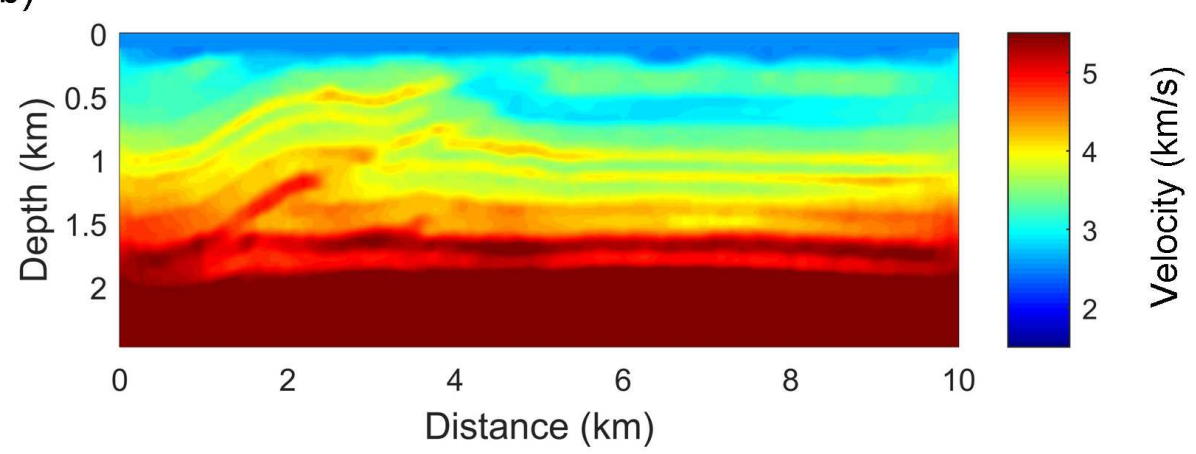

Figure 8. The inverted velocity using EWI (a), and SIEWI (b) after one sweep over the all frequencies with one iteration per frequency with the wrong wavelet. 


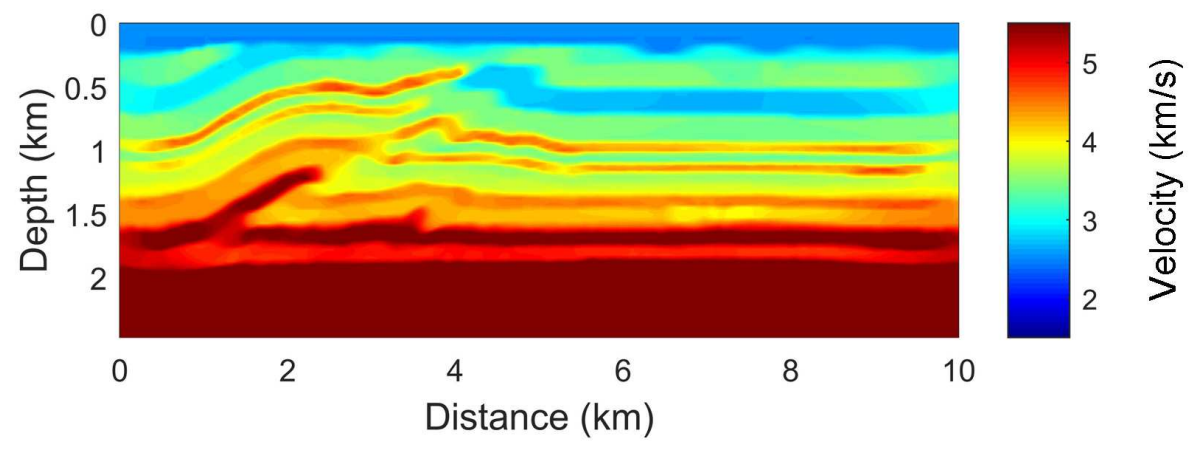

Figure 9. The inverted velocity using SIEWI after five outer iterations with the wrong wavelet.

Alkhalifah 2020b). The utilization of outer iterations in frequency domain waveform inversion implementations is sometimes needed to further cement the data fitting for low frequencies after including updates from the high frequencies (Pratt 1999). The outer iterations involve repeating EWI from low to high frequencies once again starting from the velocity model obtained in the previous EWI sweep. Since, in our EWI implementation, we update the velocity once per frequency, the outer iterations are expected to add additional information as the velocity model matures. The outer iteration also can be used to include more data in the inversion as we can include frequencies in the second sweep not used in the first sweep. The inner and outer loops are demonstrated in Algorithm 1.

\section{EXAMPLES}

In this section, we show results from synthetic data generated from the Overthrust model and a modified Marmousi model. Finally, we demonstrate the effectiveness of the proposed approach on field data from Western Australia.

\subsection{Overthrust model}

We apply the proposed method on the Overthrust model. The true velocity is shown in Fig. 11a, and the initial velocity we use is a highly smoothed version of the true velocity shown in Fig. 1 1 . The true time-domain wavelet is shown in Fig. 2 a. The peak frequency of the Ricker wavelet is $10 \mathrm{~Hz}$, and its frequency spectrum is shown in Fig. 20. We transform the time-domain wavelet using fast Fourier transform (FFT) to generate synthetic frequency-domain data. In practical FWI implementations, the true wavelet information is usually unavailable, so we might end up using a wrong wavelet such as the one in Fig. $3 \mathrm{a}$ to perform the wavefield reconstruction and inversion. The frequency spectrum of the wrong wavelet is shown in Fig. $3 \mathrm{p}$, and we can easily notice the phase distortion and amplitude change compared to the frequency spectrum of the original wavelet. The key point of wavefield reconstruction-based methods is to invert for a good wavefield at the beginning. Figs. 4 a and 40

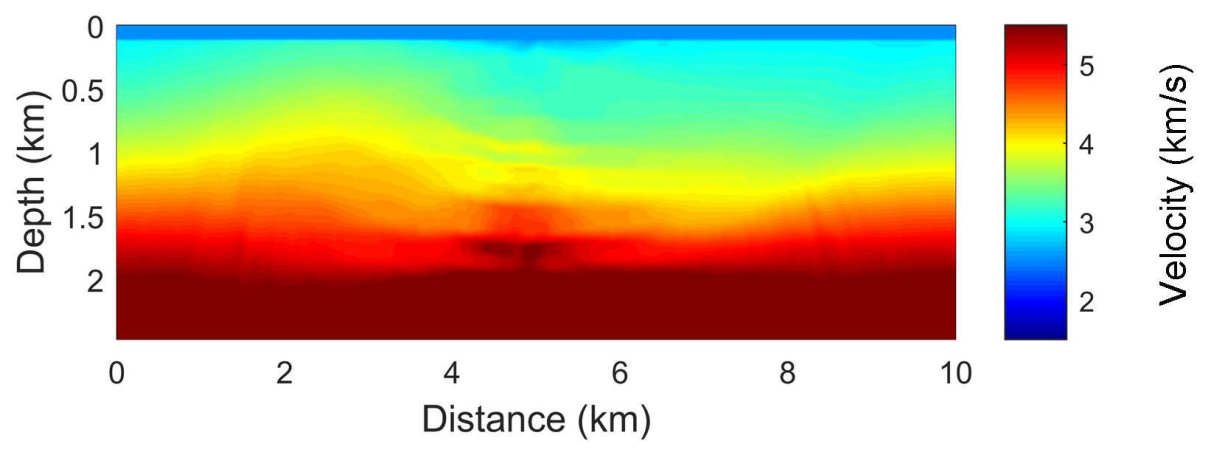

Figure 10. The inverted velocity using SIEWI after one sweep over the all frequencies and one iteration per frequency with the wrong wavelet and reference traces at a fixed receiver location. 
(a)

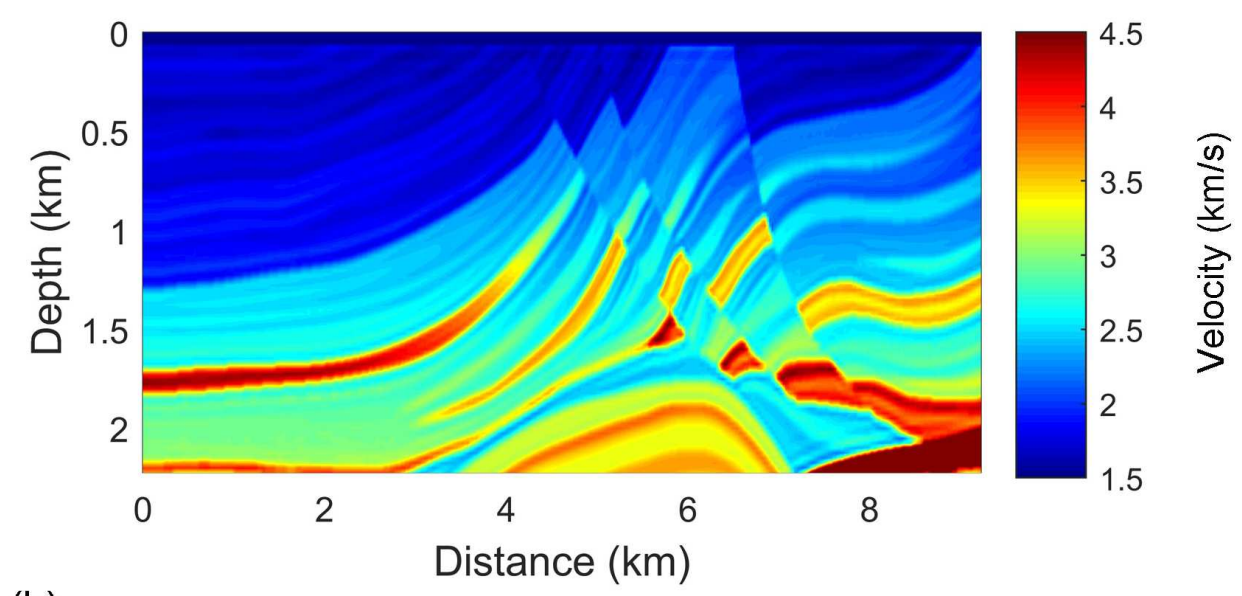

(b)

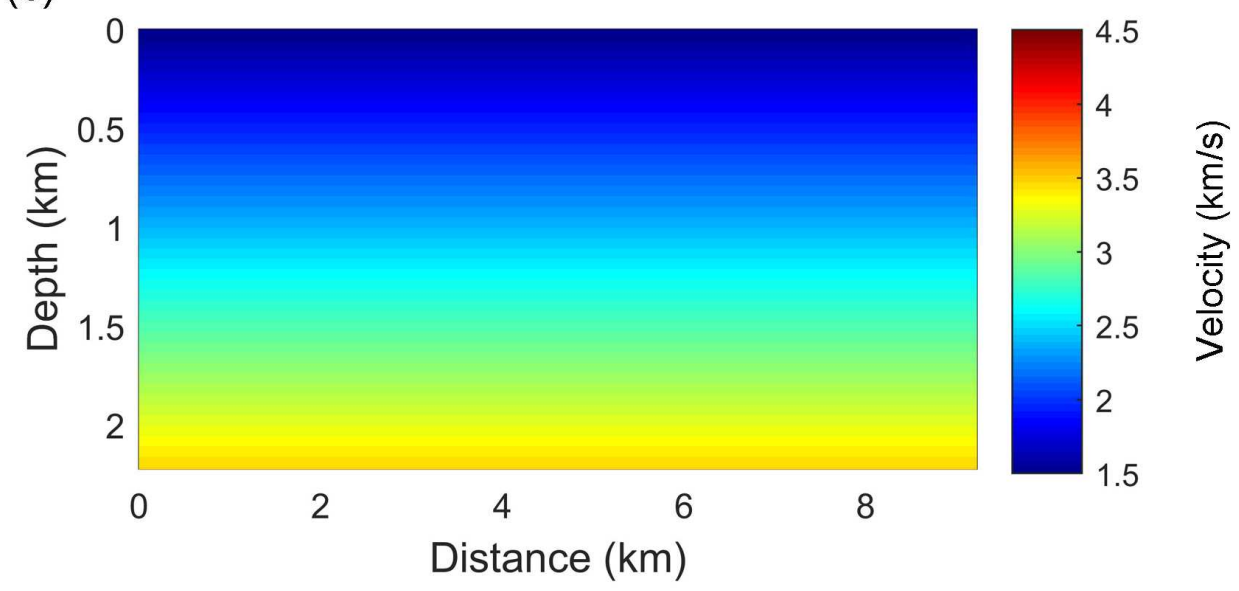

Figure 11. The true (a), and initial (b) modified Marmousi models.

are true wavefields calculated using the wave equation with the true and initial velocities, respectively, at $4 \mathrm{~Hz}$. If we use the augmented wave equation 5 to invert the wavefield using the wrong wavelet and the initial velocity, the inverted wavefield is shown in Fig. 4k. As the black arrow points out, we see that there is obvious polarity change near the source location. By comparison, the wavefield calculated by the source-independent augmented wave equation using equation 11 is shown in Fig. $4 \mathrm{~d}$, and it is obvious that no polarity change exists near the source location. 
(a)

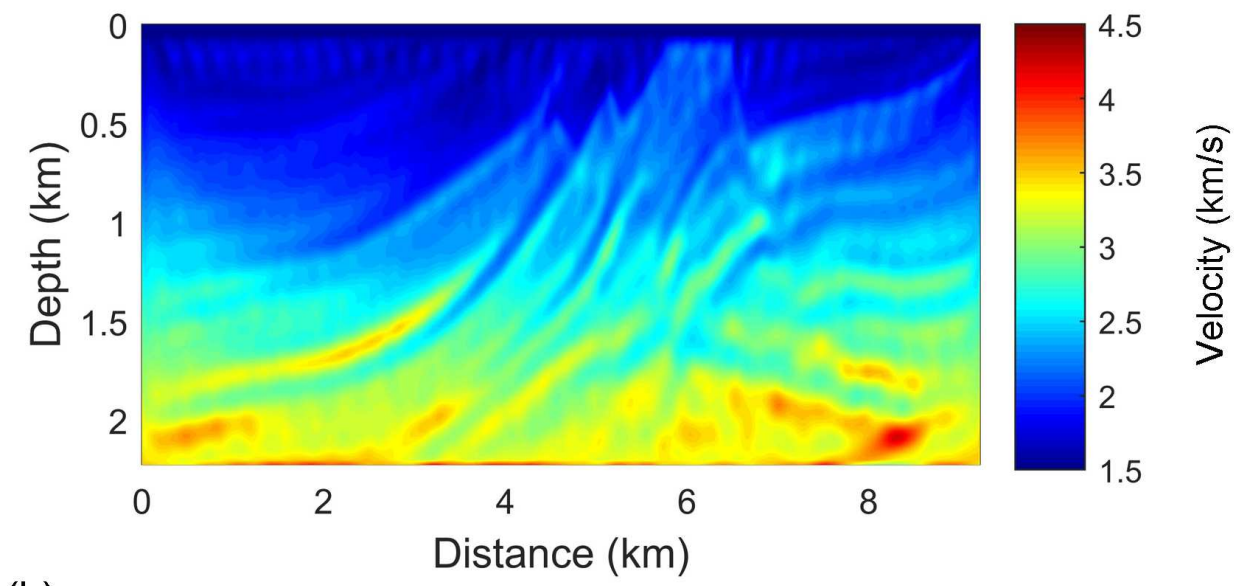

(b)

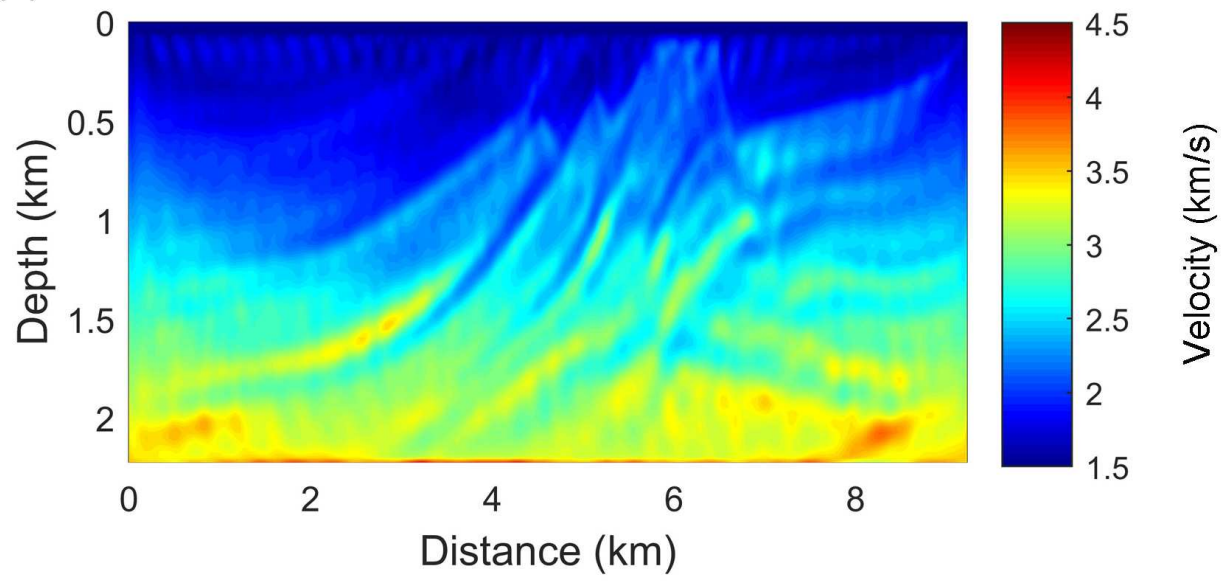

Figure 12. The inverted velocity using WRI (a), and FWI (b) with the true wavelet after one sweep over the all frequencies with one iteration per frequency.

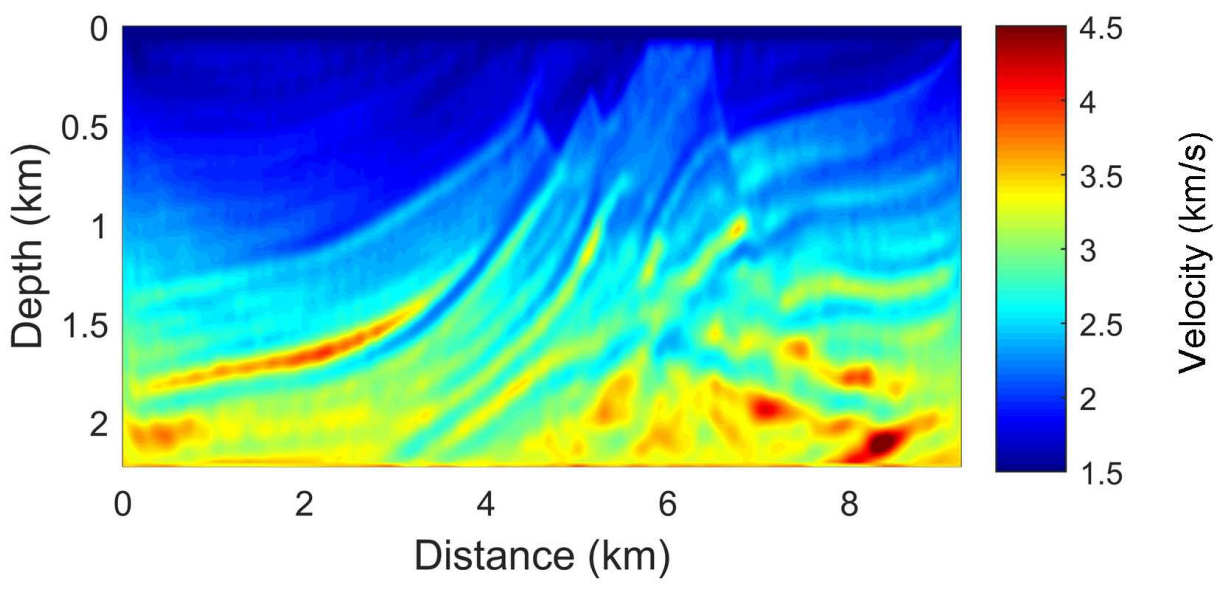

Figure 13. The inverted velocity using EWI with the true wavelet after one sweep over the all frequencies with one iteration per frequency. 
(a)

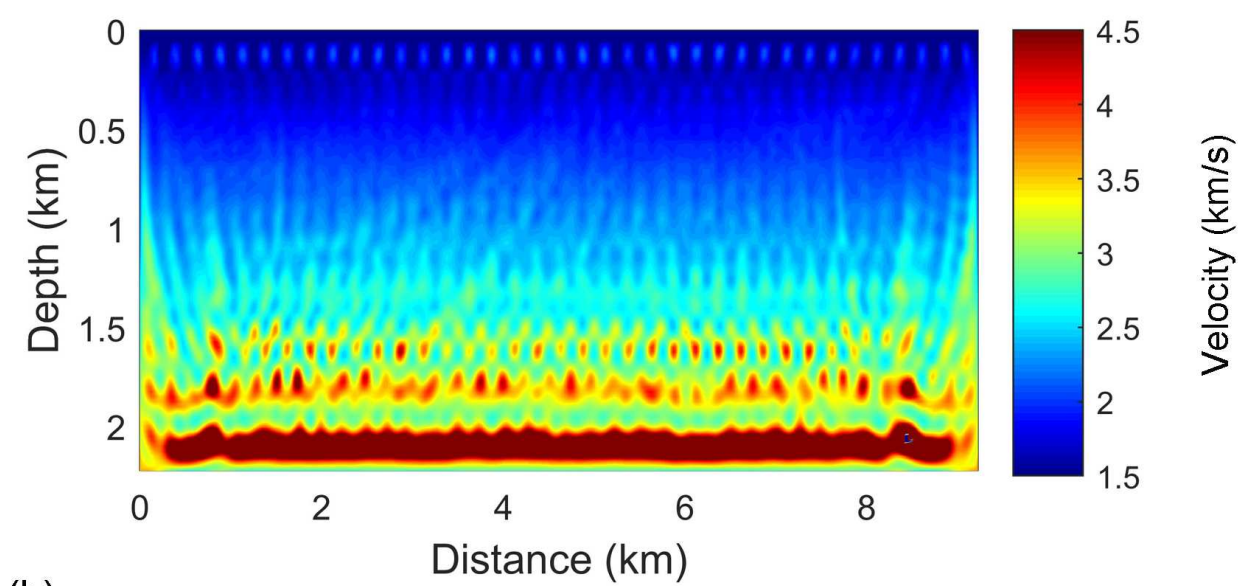

(b)

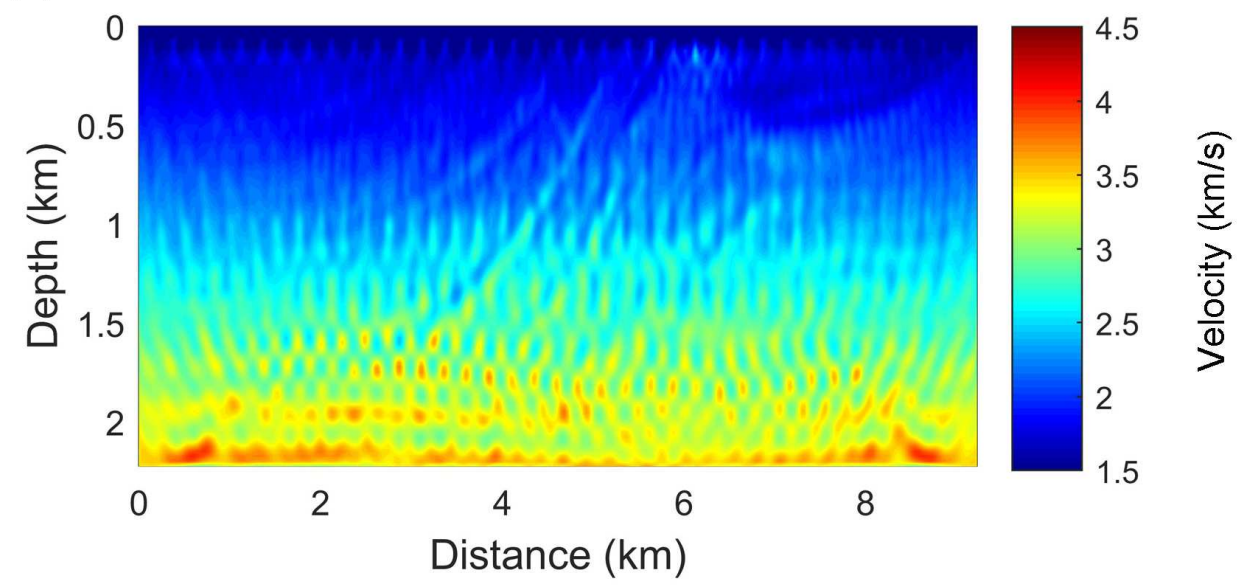

Figure 14. The inverted velocity using WRI (a), and FWI (b) with the wrong wavelet after one sweep over the all frequencies with one iteration per frequency. 
(a)

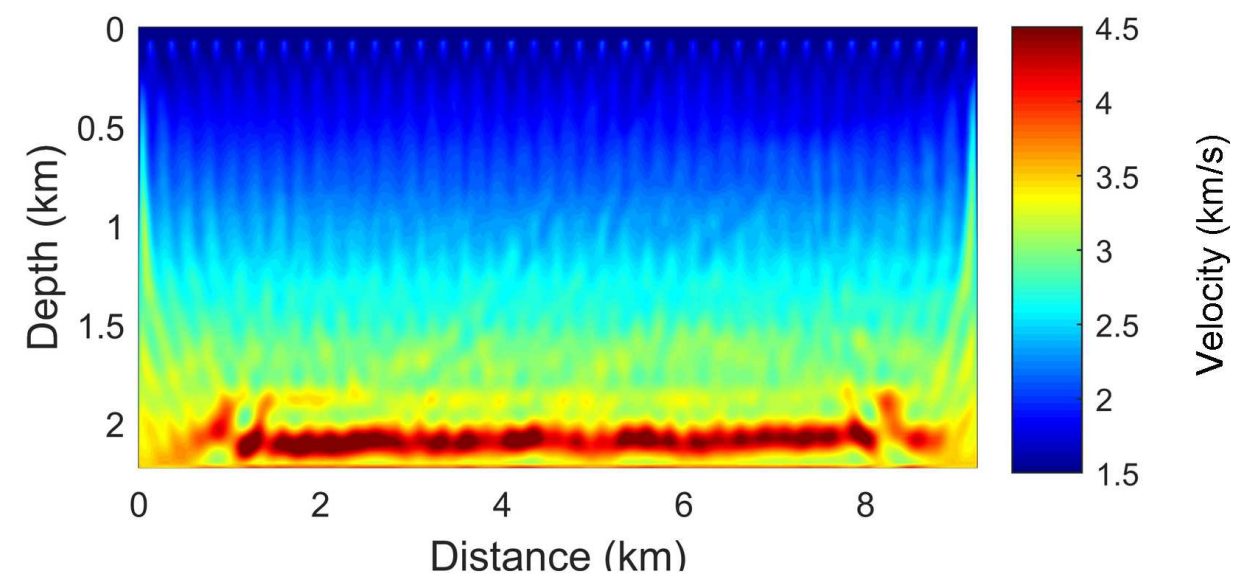

(b)

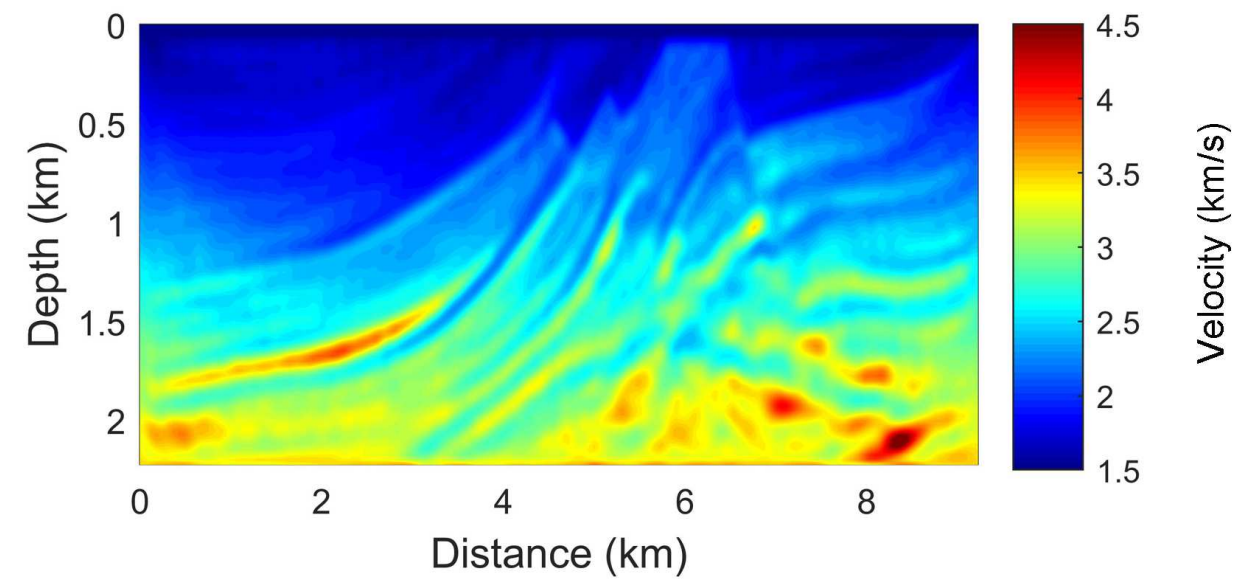

Figure 15. The inverted velocity using EWI (a), and SIEWI (b) with the wrong wavelet after one sweep over the all frequencies with one iteration per frequency.

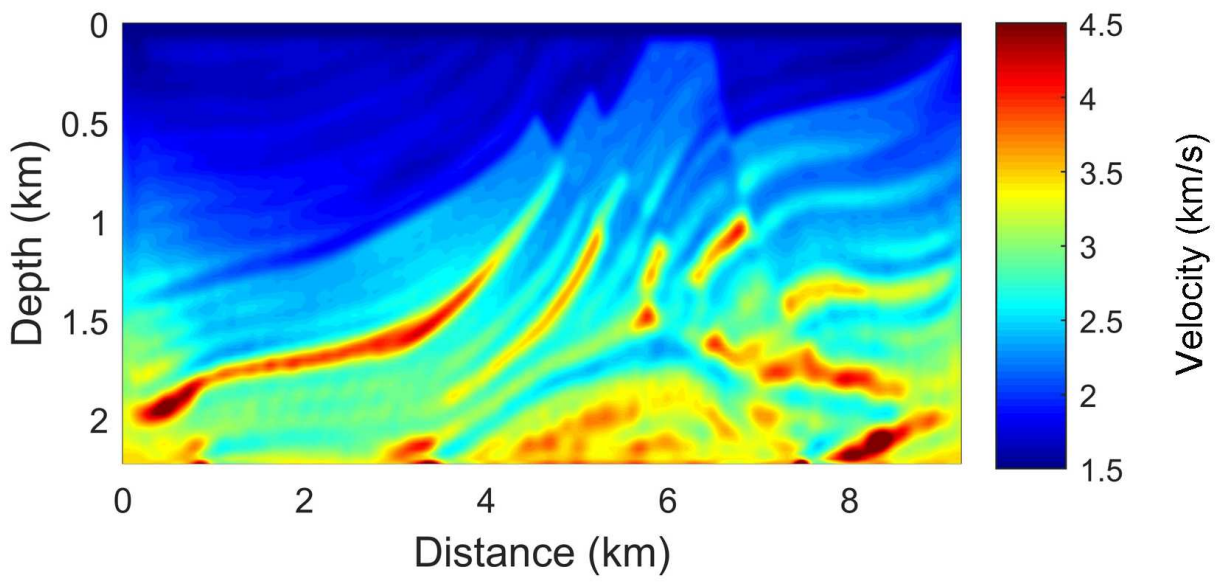

Figure 16. The inverted velocity using SIEWI with the wrong wavelet after five outer iterations. 
(a)

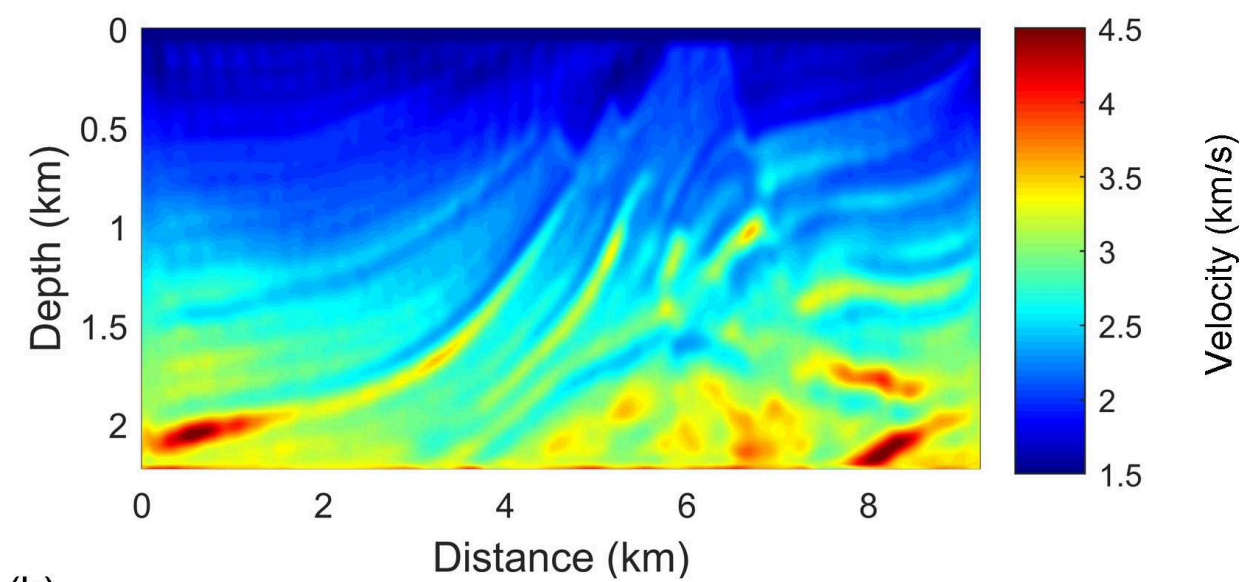

(b)

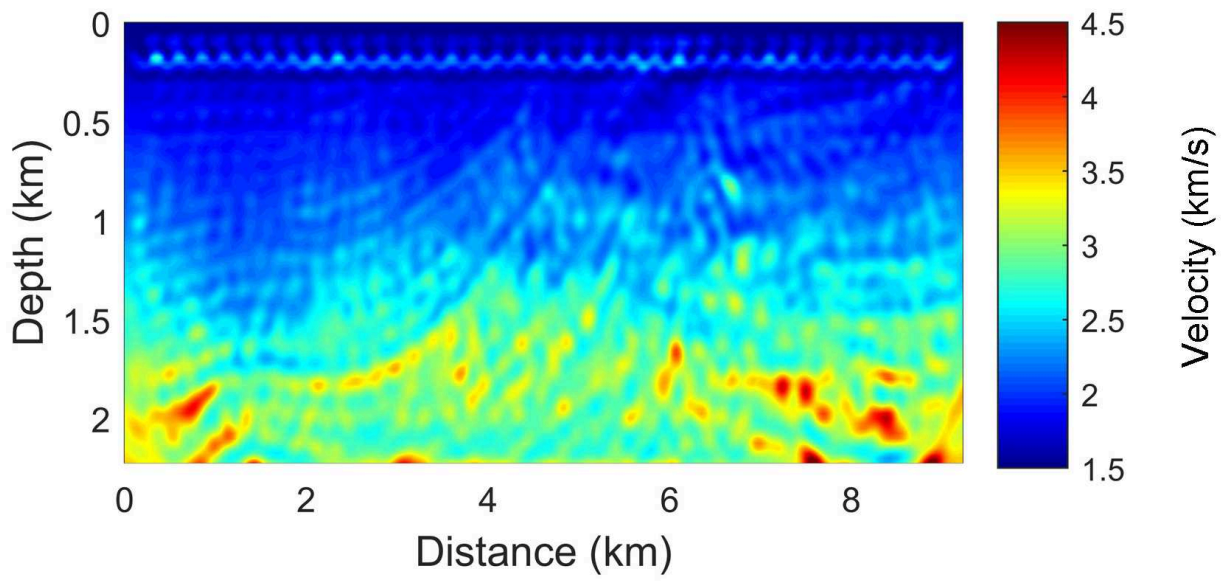

Figure 17. The inverted velocity using WRI (a), and FWI (b) with source estimation after five outer iterations.

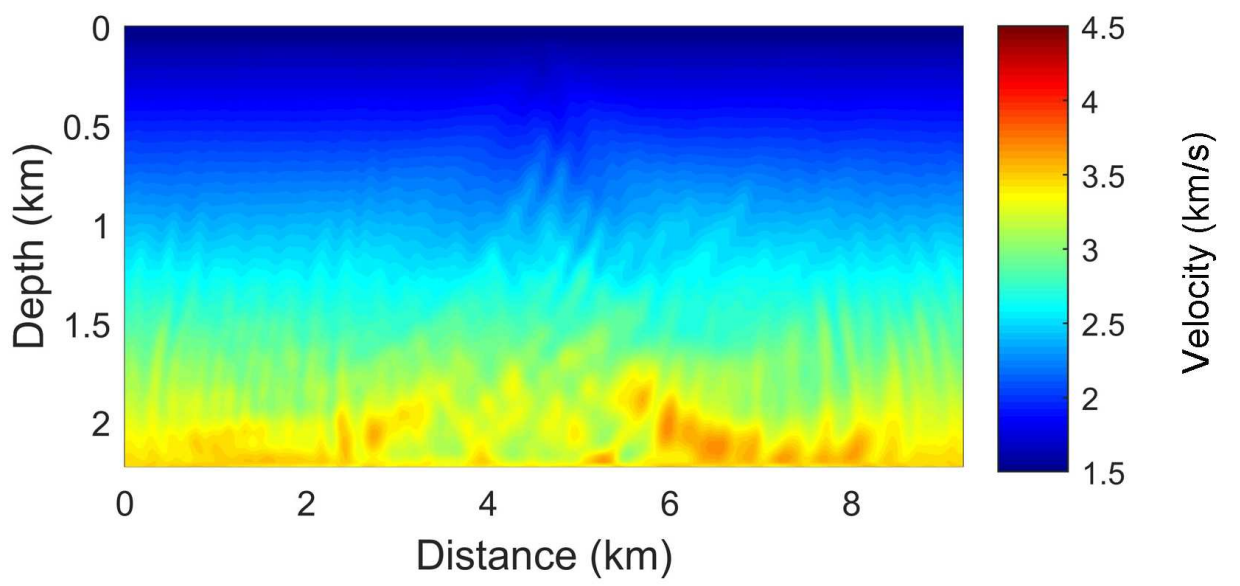

Figure 18. The inverted velocity using SIEWI after one sweep over the all frequencies with one iteration per frequency using the wrong wavelet and reference traces at a fixed receiver location. 
(a)

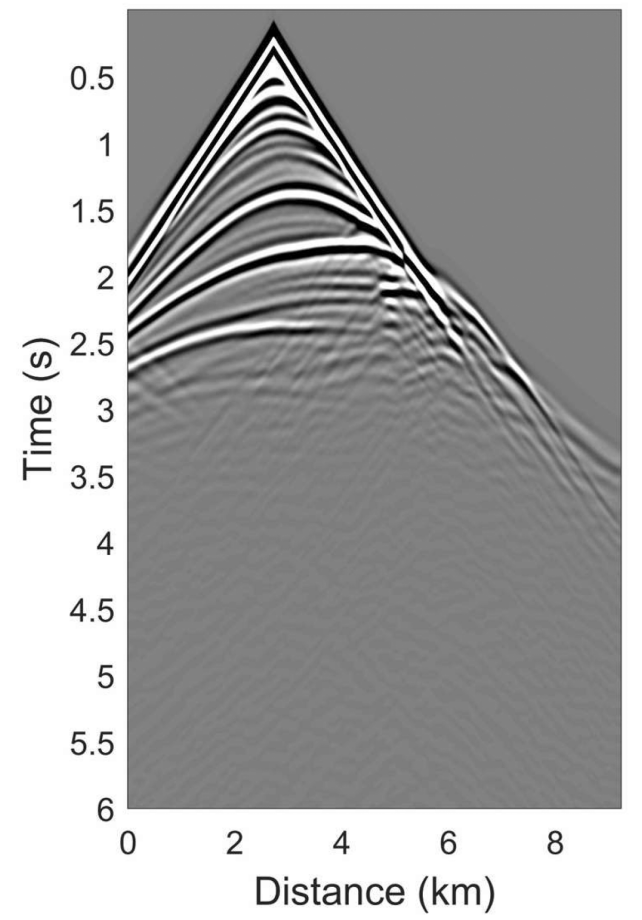

(b)

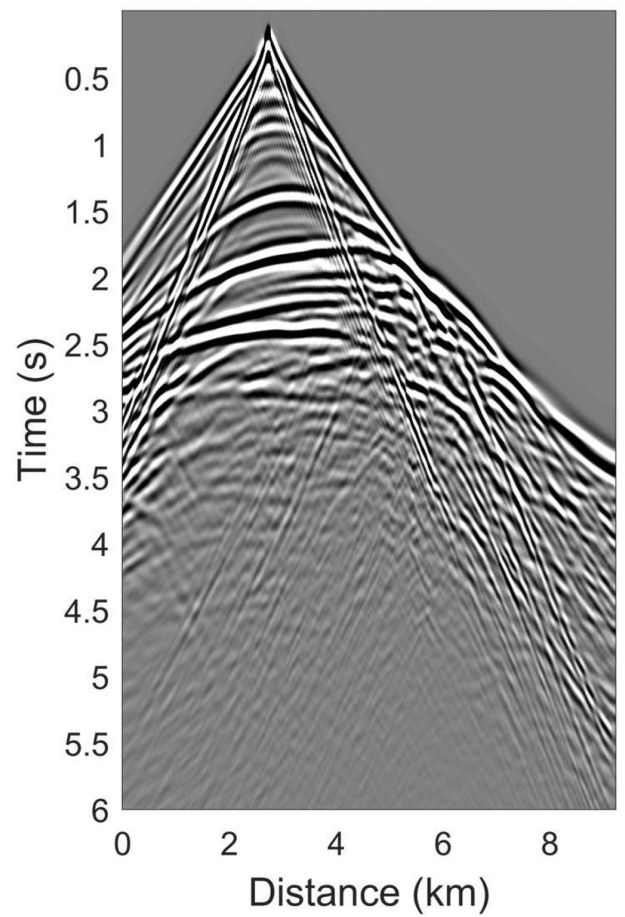

Figure 19. One shot gather at the distance of $2.75 \mathrm{~km}$ from the time-domain data using an acoustic (a), and an elastic (b) wave equation solver corresponding to the true velocity.

(a)

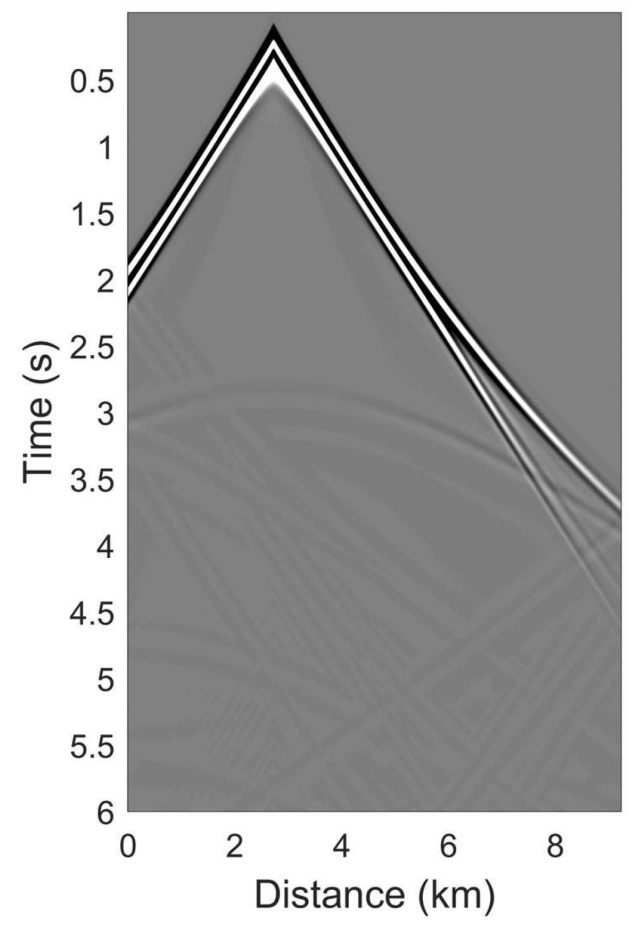

(b)

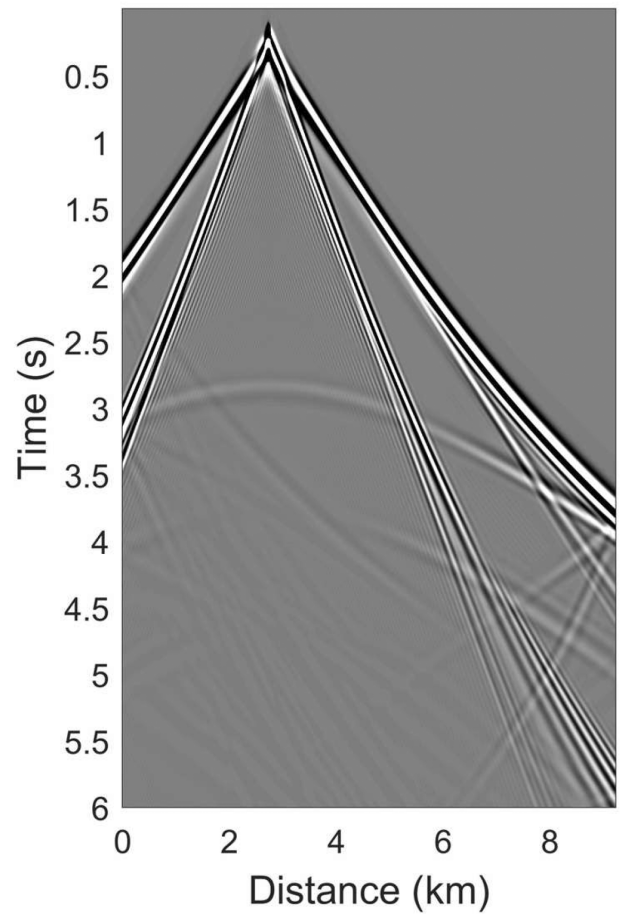

Figure 20. One shot gather at the distance of $2.75 \mathrm{~km}$ from the time-domain data using an acoustic (a), and an elastic (b) wave equation solver corresponding to the initial velocity. 
(a)

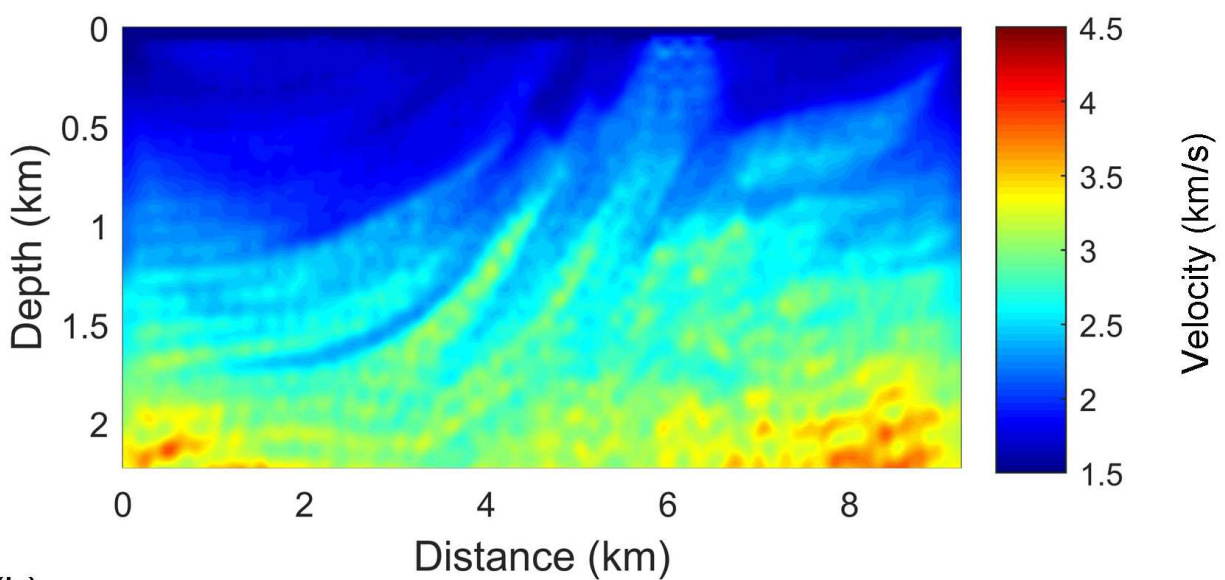

(b)

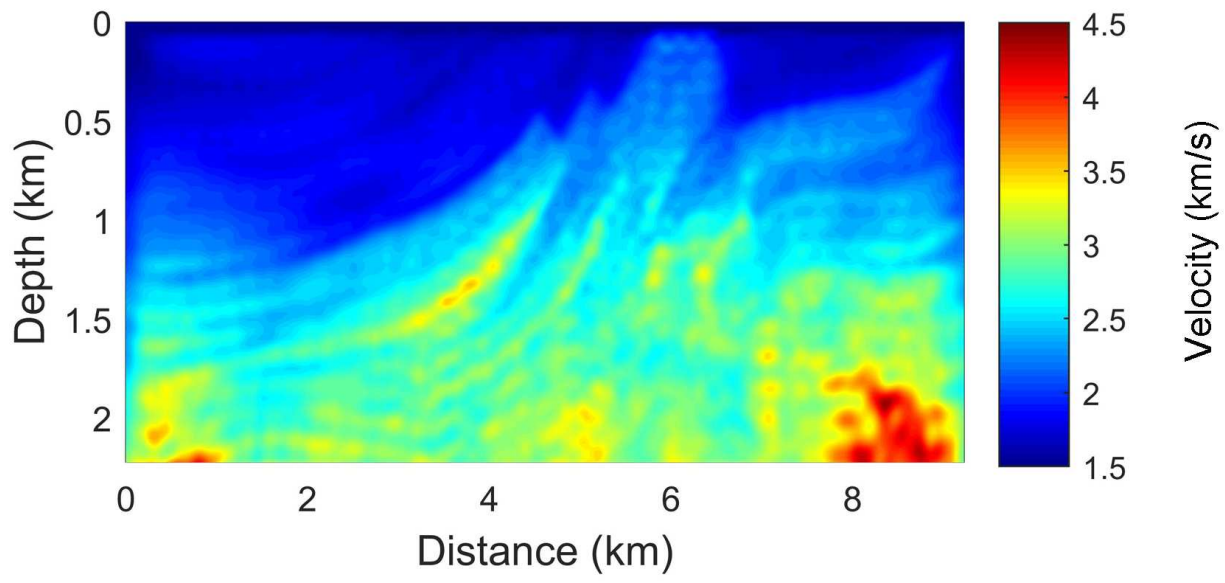

Figure 21. The inverted velocity using SIEWI after one (a), and five (b) outer iterations without the inverse crime. 
(a)

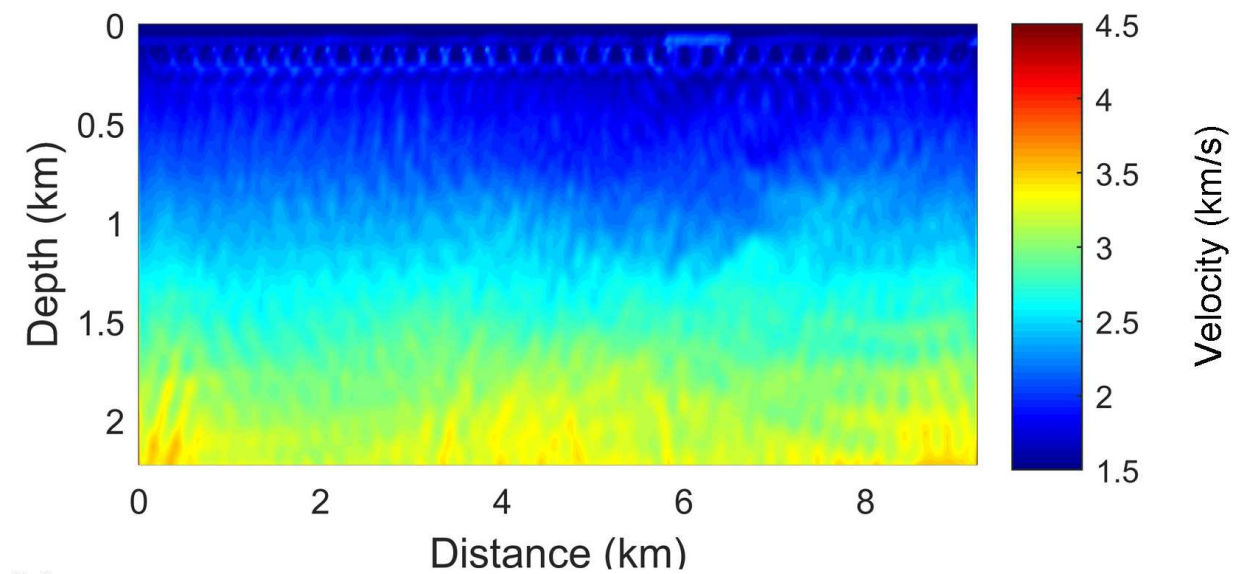

(b)

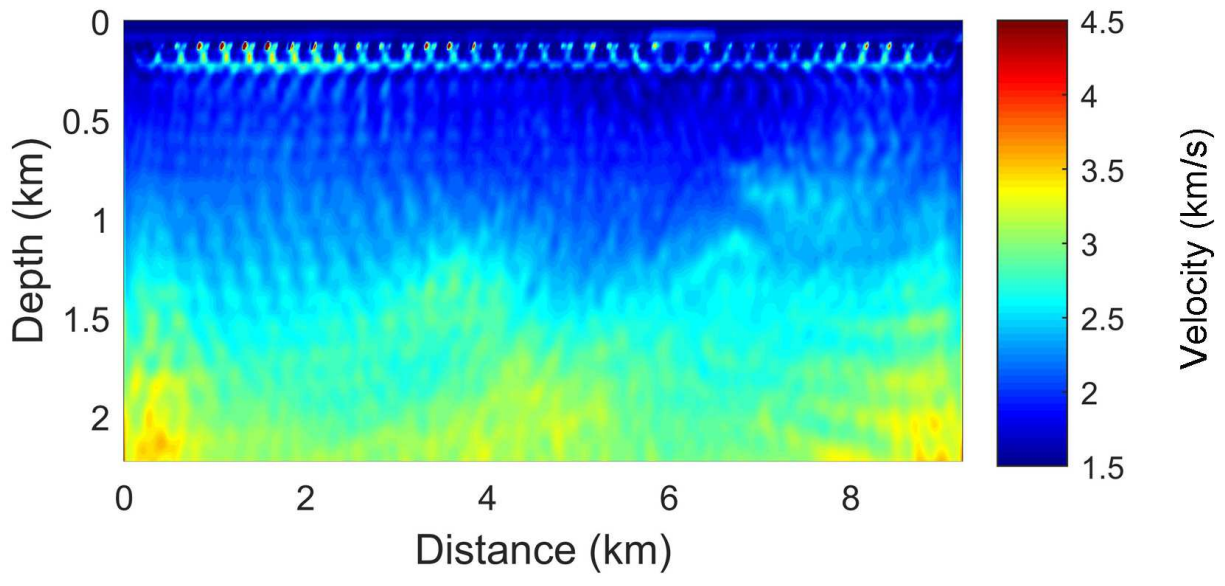

Figure 22. The inverted velocity using FWI after one (a), and five (b) outer iterations without the inverse crime.

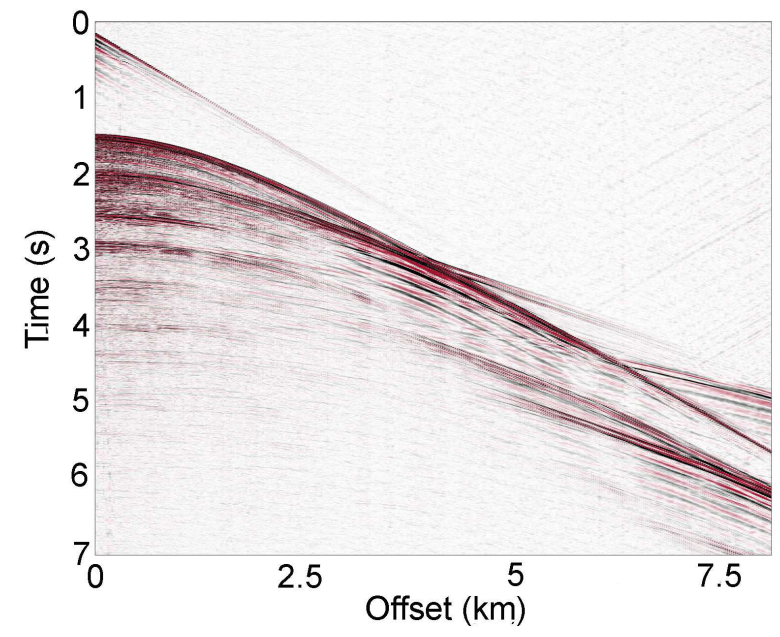

Figure 23. One shot gather from the real data at the distance of $4.6 \mathrm{~km}$. 


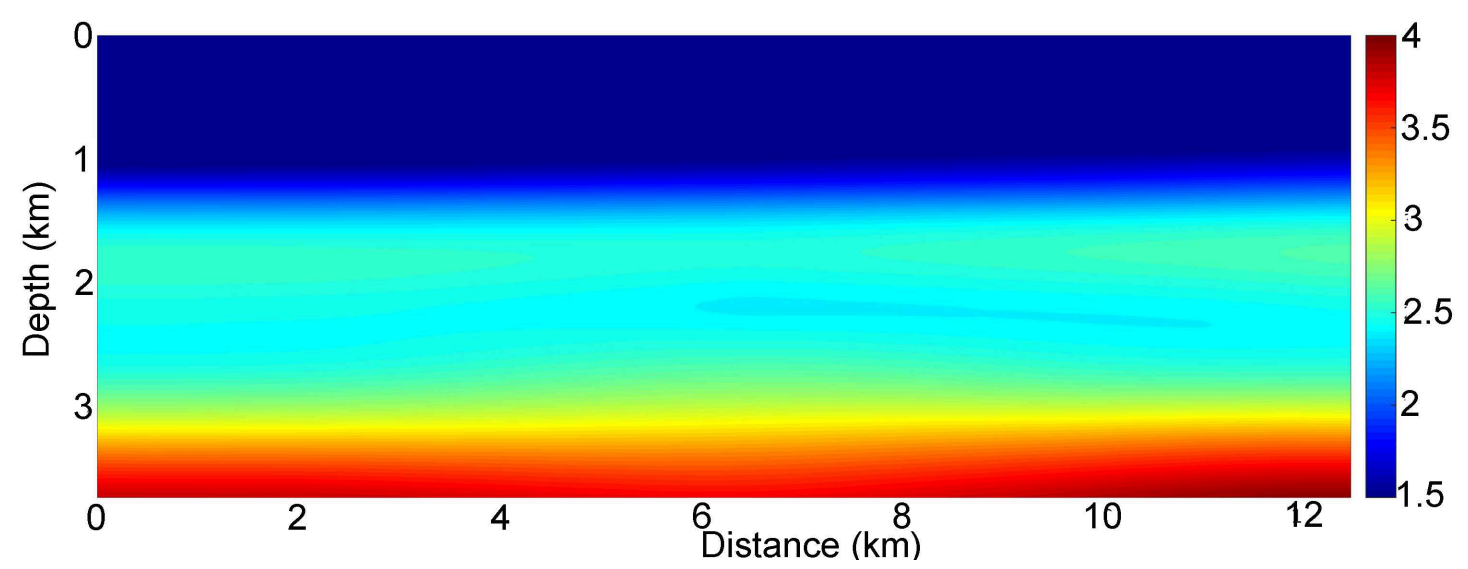

Figure 24. The initial velocity.

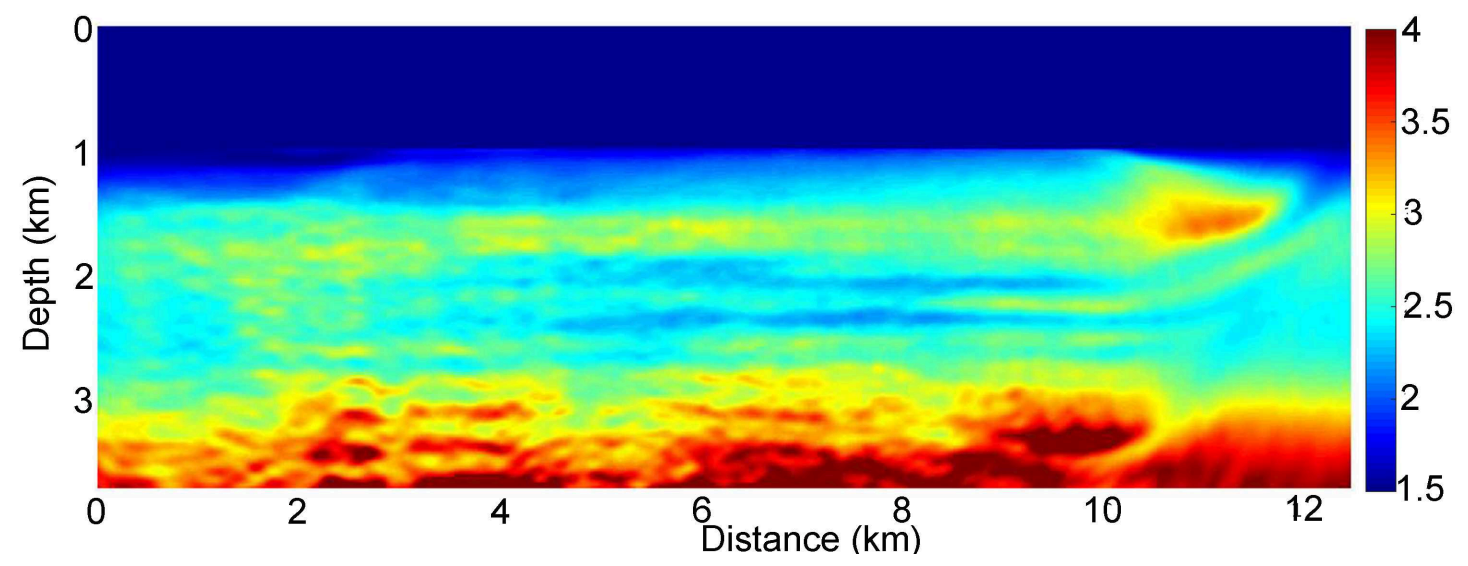

Figure 25. The inverted velocity using SIEWI.

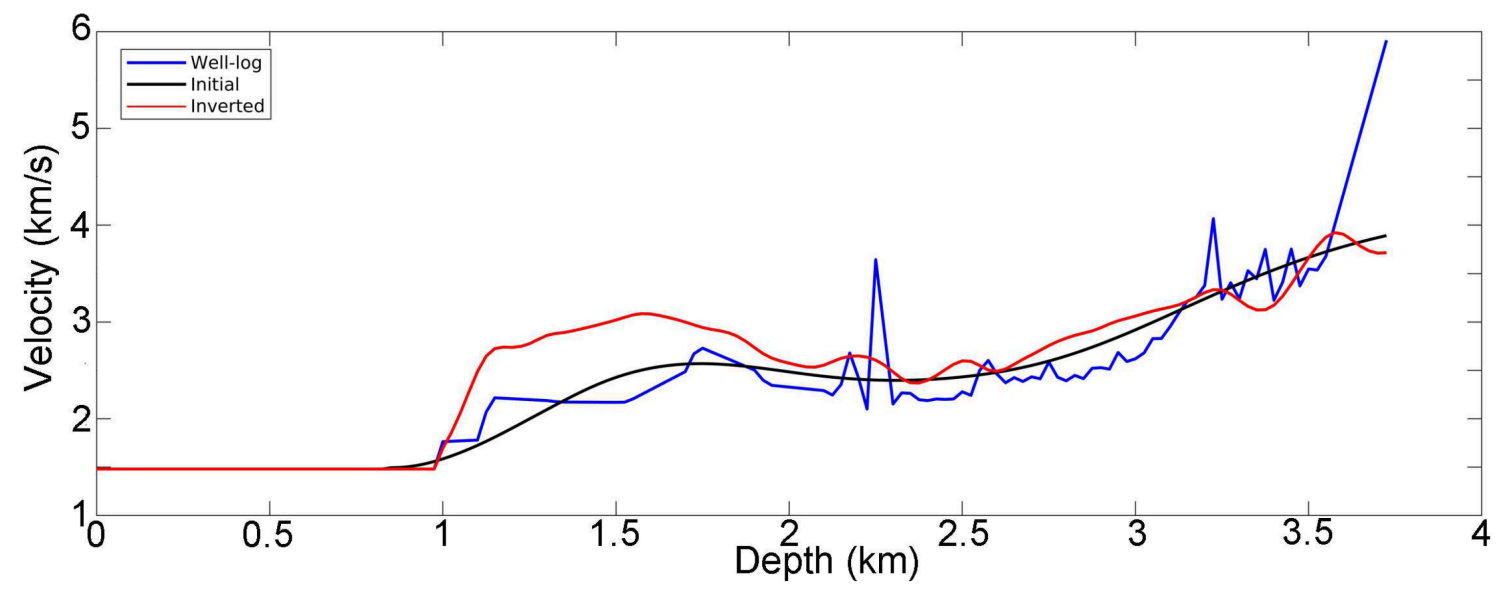

Figure 26. The velocity profile at the distance of $10.5 \mathrm{~km}$. 


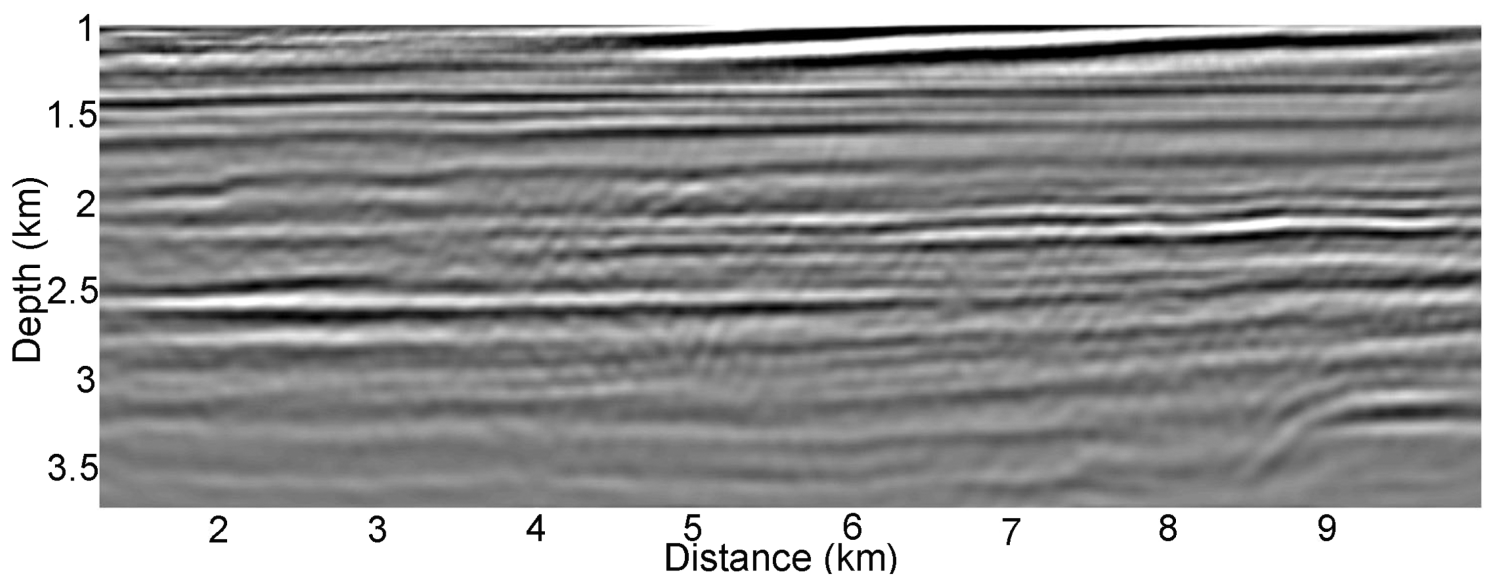

Figure 27. The RTM image from the initial velocity.

We use 21 sources uniformly distributed on the surface, and all the grid points on the surface act as receivers. Receivers' positions are fixed like ocean botton cable (OBC) acquisition. We transform the true wavelet shown in Fig. 2 a into the frequency domain, and generate the true data ranging from $3 \mathrm{~Hz}$ to $15 \mathrm{~Hz}$ with a sampling interval of $0.5 \mathrm{~Hz}$. The $\epsilon^{2}$ in this example is set $10^{7}$. A detailed study on the proper epsilon is given in Alkhalifah \& Song (2019). We first perform a standard WRI and FWI using the accurate wavelet. After one iteration through the whole frequency band, the inverted results are shown in Figs. 5a and 5p for WRI and FWI, respectively. Fig. 6 shows the inversion result using EWI. As the initial model is reasonably good, all the three methods reconstruct the main structures in the true model. If we use the wrong wavelet to repeat the inversion, the results using WRI and FWI are shown in Figs. $7 \mathrm{a}$ and 7b, respectively. We observe that both methods fail to get a reasonable model. Using the wrong wavelet, the inverted velocity using EWI after one sweep over the all frequencies with one iteration per frequency is shown in Fig. 8 a. There are strong source-signature artifacts in the shallow part of the inverted model. Using SIEWI, the inversion result is shown in Fig. 8 $\mathrm{b}$. We can see that the proposed method recovers detailed structure of the true model even with one iteration over all frequencies. After five outer iterations, the inverted velocity using SIEWI is shown in Fig. 9, and all structures in the Overthrust model are reasonably inverted with high resolution.

In SIEWI, we need to choose reference traces from both observed and predicted data. In the examples shown above, we choose the reference trace closest to the source location for both observed and predicted data, and this means we use reference traces at different receiver locations for different shot gathers. If we choose a reference trace at a fixed receiver location (receiver at the middle of the model) for all the shot gathers, the inversion result is shown in Fig. 10. Although there are no strong artifacts, no obvious update exists in the model.

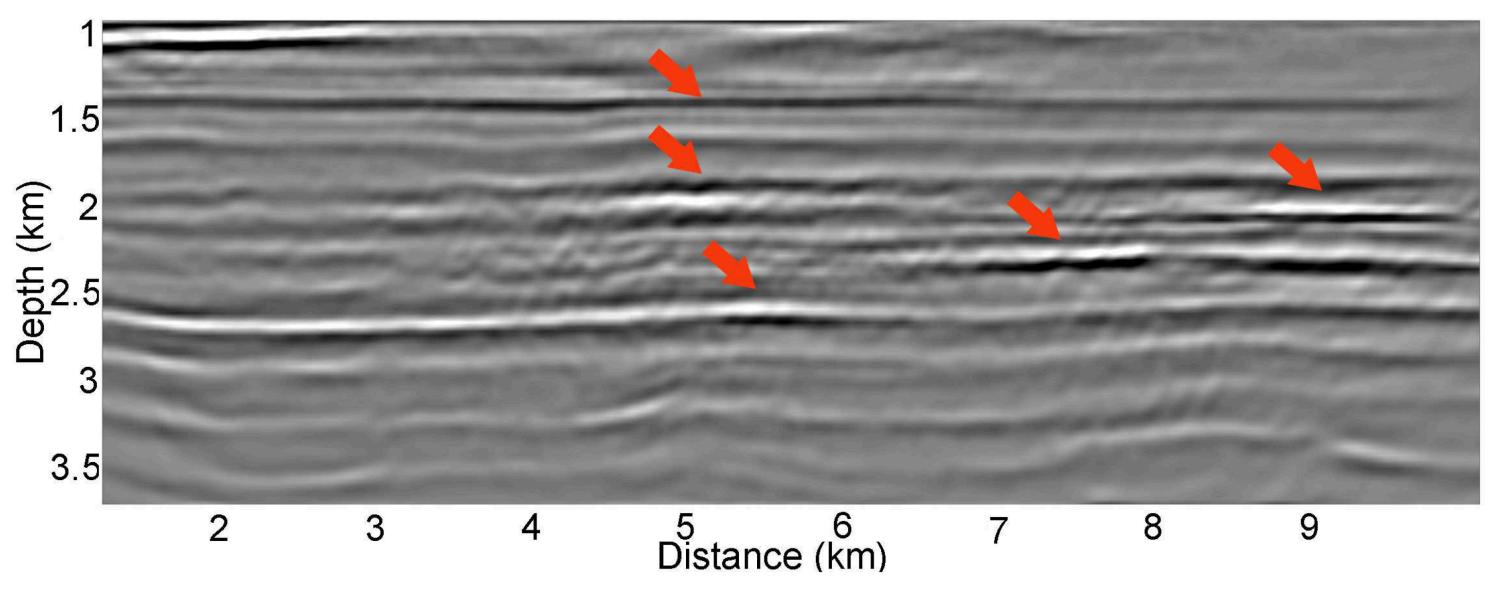

Figure 28. The RTM image from the inverted velocity. 


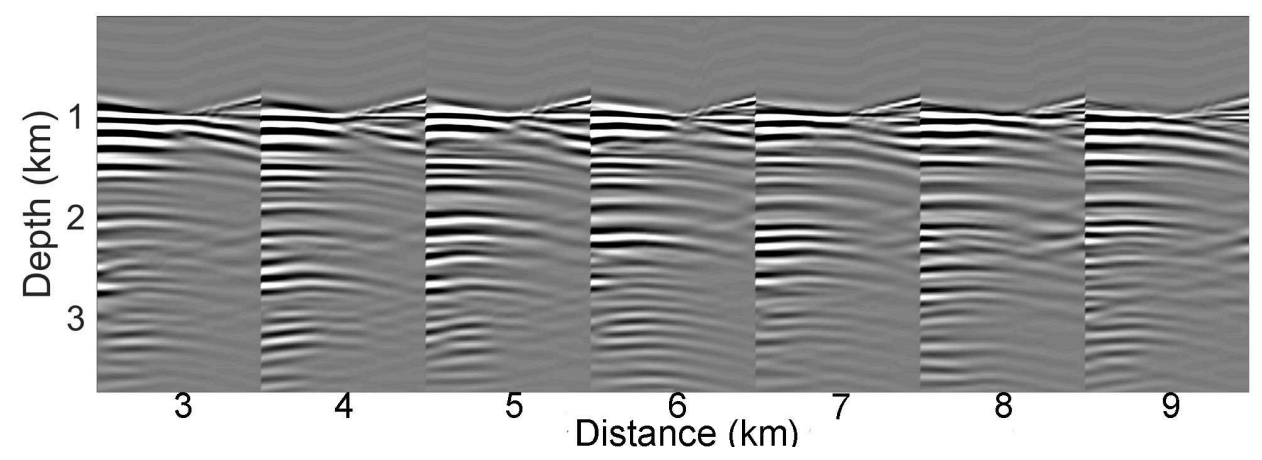

Figure 29. ADCIGs at $x=\{3,4,5,6,7,8,9\} \mathrm{km}$ corresponding to the initial velocity.

\subsection{Marmousi model}

We further apply SIEWI on a modified Marmousi model, which is shown in Fig. 11 1 . The initial velocity we use is linearly increasing with depth, which is shown in Fig. $11 \mathrm{p}$. There are 36 sources with $250 \mathrm{~m}$ spacing interval and 370 receivers fixed evenly distributed on the surface. We still use the wavelet in Fig. 2 a to generate the observed data. The frequency band we use in this example is from $4 \mathrm{~Hz}$ to $10 \mathrm{~Hz}$, and the sampling interval is still $0.5 \mathrm{~Hz}$. The $\epsilon^{2}$ we use here is also $10^{7}$. If we perform the WRI and FWI after one sweep over the all frequencies with one iteration per frequency, the inverted velocity models are shown in Figs. $12 \mathrm{p}$ and $12 \mathrm{p}$, respectively. The structures of the true model are recovered more or less by both two methods, but they clearly suffer from the cycle skipping issue. Using the same inversion setup and strategy, EWI can recover more details of the true model due to utilizing the multiscattering components in the reconstructed wavefields Alkhalifah \& Song 2019), which is shown in Fig. 13 If we use the wrong wavelet (Fig. 3a) to repeat the inversion process using WRI and FWI, we end up with the inverted models shown in Fig. 14a and 14 b, respectively. It is obvious that using the wrong wavelet, we cannot get reasonable inversion results. Fig. 15 a shows the inversion result using EWI with the wrong wavelet. We observe that a wrong wavelet will be a more severe problem for wavefield reconstruction based methods, including WRI and EWI. For EWI, there are strong artifacts near the source location. In SIEWI implementation, we still first use both reference traces at the source location. By comparison, SIEWI is able to invert for a reasonable velocity model after one sweep over the all frequencies with one iteration per frequency, which is shown in Fig. 15b. After another four outer iterations, the final inverted velocity model is shown in Fig. 16 It is obvious that the proposed method can recover a high-resolution velocity model regardless of the wavelet accuracy. Besides the source-independent based objective functions, we can also perform a source estimation step within the inversion process. With a source estimation step in WRI and FWI (Fang \& Herrmann 2015. Pratt 1999), the inversion results after five outer iterations are shown in Figs. $17 \mathrm{a}$ and $17 \mathrm{~b}$, respectively. Even with a source estimation step, FWI still fails to recover a reasonable velocity model. Though WRI benefits a lot from the source estimation, it is still not comparable to SIEWI. To demonstrate the importance of the reference trace selection, we show the inversion result with a reference trace at a fixed receiver location (receiver at the middle of the model) after one sweep over the all frequencies with one iteration per frequency in Fig. 18. There is barely any useful information getting recovered in the model compared to the result from a dynamic reference trace near the source location.

In order to avoid committing the inverse crime and demonstrate the validity of the proposed method in more realistic

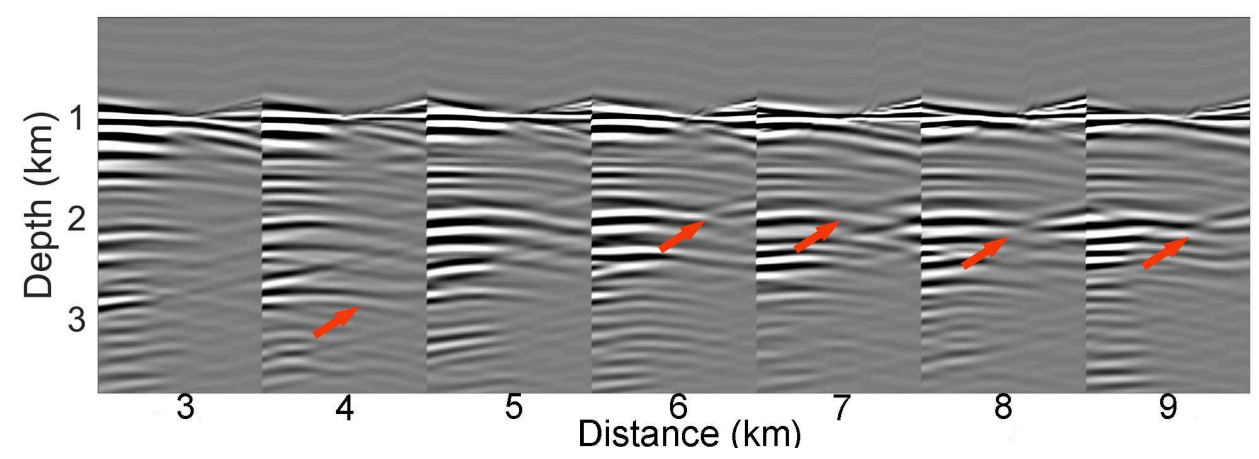

Figure 30. ADCIGs at $x=\{3,4,5,6,7,8,9\} \mathrm{km}$ corresponding to the inverted velocity. 


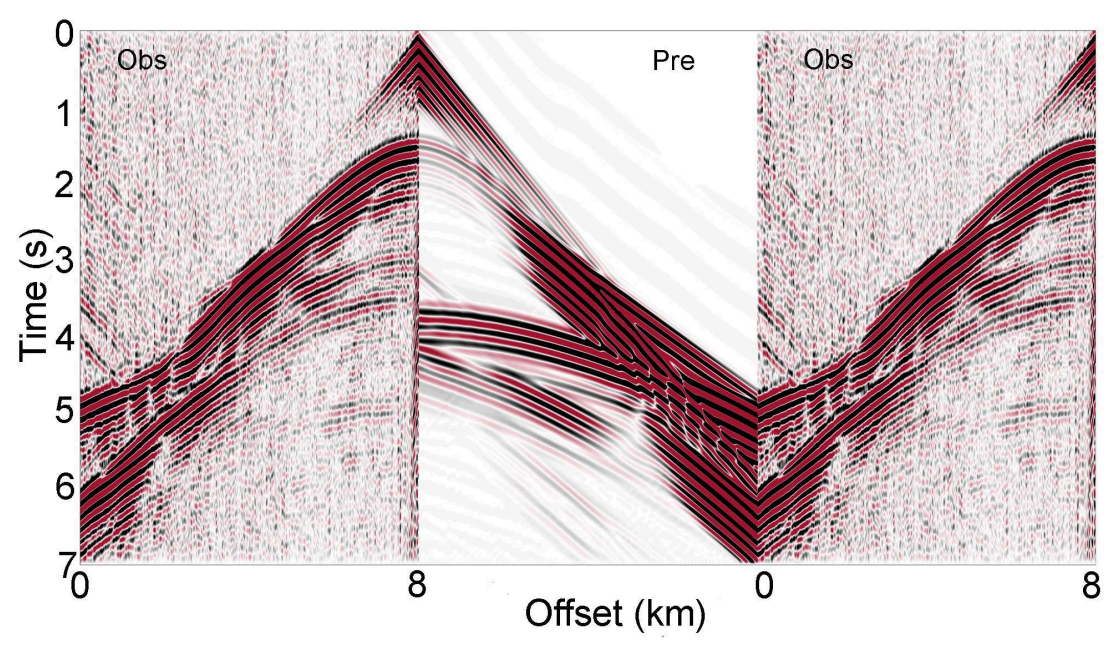

Figure 31. Filtered observed data (left, right) at $4.6 \mathrm{~km}$, and corresponding predicted data (middle) using the initial velocity.

cases, we generate the observed data using a time-domain acoustic wave equation and elastic wave equation solver with an explosive source. We use a time-domain Ricker wavelet to generate the data. One shot gather from the acoustic data and the z-component elastic data at the distance of $2.75 \mathrm{~km}$ are shown in Figs. 19a, and $19 \mathrm{p}$, respectively. We also show one shot gather from the acoustic data and the z-component elastic data at the same location corresponding to the initial velocity in Figs. 20 , and 20 , respectively. The absence of reflection waves indicates that the initial velocity model is smooth and generally crude. Clearly, we see that the z-component of the elastic data contains surface waves and S-wave artifacts. Thus, we use this z-component of the elastic data in our acoustic inversion. We build the S-wave velocity the same way as previous by dividing it by a scalar, and use a constant density. We use the same inversion setup as what we used before in this example. The initial source function used to reconstruct the wavefield in all frequencies is Delta function. Figs. 21p and 21p show the inversion results after one and five outer iterations (sweep from low to high frequency) using SIEWI. We observe that the main structures of the Marmousi model are reasonably recovered, especially in the shallow part. In this example, we find that wavefield reconstruction based methods (WRI and EWI) are more vulnerable to the wrong source function, so we repeat the no inverse crime inversion using FWI with the same inversion setup. The inversion results after one and five outer iterations are shown in Figs. 22 and $22 \mathrm{~b}$, respectively. It is obvious that FWI fails to recover a reasonable velocity model, and strong artifacts are shown in the shallow part.

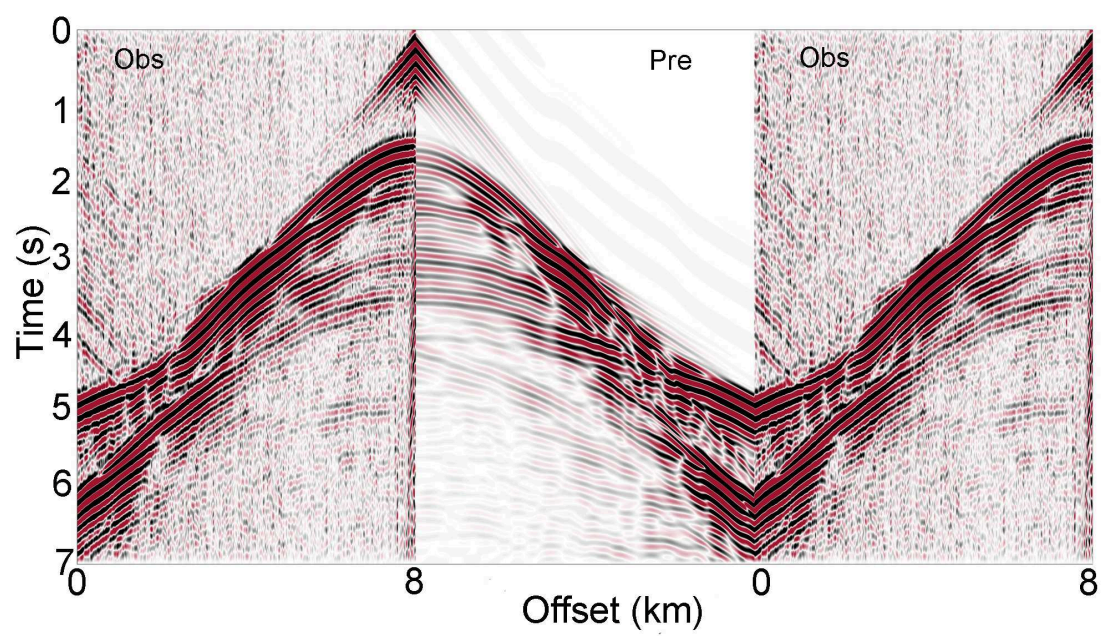

Figure 32. Filtered observed data (left, right) at $4.6 \mathrm{~km}$, and corresponding predicted data (middle) using the inverted velocity. 


\subsection{CGG Field data}

The 2D real data we use is acquired by CGG using a Broadseis acquisition system from North-Western Australia Continental Shelf (Soubaras \& Dowle 2010). Frequencies below $2.5 \mathrm{~Hz}$ has been filtered out because of the low signal-to-noise ratio. Fig. 23 shows one shot gather of the real data at the distance of $4.6 \mathrm{~km}$. The original data set contains 1824 shot gathers with an interval of $0.01875 \mathrm{~km}$, and we choose 100 shot gathers to cover the $2 \mathrm{D}$ survey area. The target survey area we focus is 12.5 $\mathrm{km}$ long and $3.75 \mathrm{~km}$ in depth, and the initial velocity model is borrowed from Kalita \& Alkhalifah (2017, 2018), as shown in Fig. 24. We resample the data and use 324 receivers from each streamer with an interval of $25 \mathrm{~m}$. This real data is collected using a marine acquisition system, and the largest offset is $8.1 \mathrm{~km}$.

Without the source wavelet estimation, we perform SIEWI from $3 \mathrm{~Hz}$ to $12 \mathrm{~Hz}$ with a dense sampling of $0.15 \mathrm{~Hz}$. The $\epsilon^{2}$ used here is set to $10^{8}$ based on our tests. The reference trace location is at the smallest offset for every shot gather. The final inversion result is shown in Fig. 25 It is obvious that the low velocity layers between $2.0 \mathrm{~km}$ to $2.5 \mathrm{~km}$ depth are well inverted. The check shot velocity from a well at $10.5 \mathrm{~km}$ is shown in Fig. 26 , and the velocity between $1 \mathrm{~km}$ and $2 \mathrm{~km}$ is obtained by interpolation. The SIEWI inverted velocity model fits the well-log velocity well below $2 \mathrm{~km}$, which demonstrates the effectiveness of SIEWI on the real data. We perform reverse time migration (RTM) using the initial and final velocities, and the images are shown in Figs. 27 and 28, respectively. As the red arrows point out, we observe multiple improvements in the RTM image using the inverted velocity. We also show the angle domain common-image gathers (ADCIGs) at locations $x=\{3,4,5,6,7,8,9\} \mathrm{km}$ ranging between 0 and $40^{\circ}$ corresponding to the initial and inverted velocity models in Figs. 29 and 30 respectively. The ADCIGs from the inverted velocity are flattened in some areas (as the red arrows point out) compared to those from the initial velocity, and these features indicate the improvement of the inverted velocity. Finally, we compare one filtered shot gather with the predicted data generated from the initial and inverted velocity models. We invert for the source wavelet in the time domain by back propagating the observed data to the source location using the inverted velocity model. From Fig. 31, we see that the predicted data using the initial velocity lacks most of the reflections apparent in observed data, and their diving waves at far offset don't match. Using the inverted model, the predicted data recovers the reflection events seen in the observed data, as shown in Fig. 32 As the kinematic features of the inverted velocity improves, the diving waves start to fit better for the observed and predicted data.

\section{DISCUSSION}

In the wavefield reconstruction based methods including WRI and EWI, $\epsilon^{2}$ balances the weight between the data and the wave equation fitting. If the initial velocity is poor, a small $\epsilon^{2}$ will reduce the cycle-skipping issue. Numerical experiments show that a single fixed value of $\epsilon^{2}$ is sufficient (Leeuwen \& Herrmann 2015). We use trial and error to find an appropriate $\epsilon^{2}$ in different scenarios. One quick way to find a proper $\epsilon^{2}$ is to use a scaling the highest eigenvalue of the auxiliary linear system Leeuwen \& Herrmann (2015). This weighting factor $\epsilon^{2}$ can also be automatically determined using a multiplicative cost function da Silva \& Yao (2017).

In EWI, for the inner iterations for updating $u$ and $f_{e}$, we start with $f_{e}=f$. In the first inner iteration, the resulting wavefield contains single scattering information to fit the data. As $f_{e}$ is updated with parameter perturbations in the model, these perturbations act as secondary sources in the following inner iterations. As a result, EWI can reconstruct a wavefield including multiscattering information by using cheap inner iterations (Alkhalifah \& Song 2019), only a few outer iterations will achieve convergence.

The source-independent objective function in equation 10 should theoretically work in combination with the original WRI. The source independence we refer to in this paper pertains to the source function (or wavelet). As EWI admits advantages in efficiency and accuracy over WRI, we directly use a source-independent EWI (SIEWI) objective function. The proposed method requires two reference traces from the observed and predicted data. From what we experienced in the examples, we find that it is better to choose the reference trace near the source location. This is because the reference trace at the near offsets contain most of the source function information, and this reference trace selection strategy is also suggested by Wang \& Alkhalifah (2018). We cannot use a referenced trace at a fixed receiver position for all the shot gathers. Choosing a reference trace at a fixed receiver location will allow us to extract less useful information of the subsurface model, and slow down the convergence rate of the optimization.

\section{CONCLUSIONS}

We proposed a source-independent efficient wavefield inversion (SIEWI) to mitigate the source wavelet effect by incorporating source-independent objective into the original EWI. If we start with a wrong source wavelet in the original EWI, the source wavelet error will propagate into the reconstructed wavefield. In this case, the theory of EWI breaks down and the optimization problem diverges from the desired solution. The proposed method removes the source wavelet accuracy dependency while 
maintaining the EWI features. Applications to synthetic data generated from the Overthrust model and a modified Marmousi model show that SIEWI is capable of obtaining reasonable inversion results regardless of the source wavelet accuracy. The test on a $2 \mathrm{D}$ field data also demonstrates the effectiveness of the proposed approach.

\section{ACKNOWLEDGEMENT}

We thank KAUST for its support and the SWAG group for the collaborative environment. This work utilized the resources of the Supercomputing Laboratory at King Abdullah University of Science and Technology (KAUST) in Thuwal, Saudi Arabia, and we are grateful for that. We also thank CGG for providing the field data set and Geoscience Australia for providing the well-log information. We thank the assistant editor, the editor, Herve Chauris, and the reviewers for their critical and helpful review of the manuscript. 


\section{REFERENCES}

Alkhalifah, T., 2019. Linear wavefield optimization using a modified source, Communications in computational physics, p. accepted.

Alkhalifah, T. \& Song, C., 2019. An efficient wavefield inversion: Using a modified source function in the wave equation, Geophysics, 84(6), R921-R934.

Alkhalifah, T. \& Wu, Z., 2016. Multiscattering inversion for low-model wavenumbers, Geophysics, 81(6), R417-R428.

Biondi, B. \& Almomin, A., 2014. Simultaneous inversion of full data bandwidth by tomographic full-waveform inversion, Geophysics, 79(3), WA129-WA140.

Chi, B., Gao, K., \& Huang, L., 2018. Source-independent full-waveform inversion using an amplitude-semblance objective function, in SEG Technical Program Expanded Abstracts 2018, pp. 1268-1272, Society of Exploration Geophysicists.

Choi, Y. \& Alkhalifah, T., 2011. Source-independent time-domain waveform inversion using convolved wavefields: Application to the encoded multisource waveform inversion, Geophysics, $\mathbf{7 6}(5)$, R125-R134.

Choi, Y., Shin, C., Min, D.-J., \& Ha, T., 2005. Efficient calculation of the steepest descent direction for source-independent seismic waveform inversion: An amplitude approach, Journal of Computational Physics, 208(2), 455-468.

Choi, Y., Min, D.-J., \& Shin, C., 2008. Frequency-domain elastic full waveform inversion using the new pseudo-hessian matrix: Experience of elastic marmousi-2 synthetic data, Bulletin of the Seismological Society of America, 98(5), 2402-2415.

da Silva, N. V. \& Yao, G., 2017. Wavefield reconstruction inversion with a multiplicative cost function, Inverse Problems, 34(1), 015004.

Fang, Z. \& Herrmann, F., 2015. Source estimation for wavefield reconstruction inversion: 77th annual international conference and exhibition, eage, Extended Abstracts, Tu N, 104, 05.

Fang, Z., Wang, R., \& Herrmann, F. J., 2018. Source estimation for wavefield-reconstruction inversion, Geophysics, 83(4), R345-R359.

Kalita, M. \& Alkhalifah, T., 2017. Efficient full waveform inversion using the excitation representation of the source wavefield, Geophysical Journal International, 210(3), 1581-1594.

Kalita, M. \& Alkhalifah, T., 2018. Multiscale full-waveform inversion using flux corrected transport, SEG Technical Program Expanded Abstracts, pp. 1153-1157.

Kamei, R., Pratt, R., \& Tsuji, T., 2013. On acoustic waveform tomography of wide-angle obs datastrategies for pre-conditioning and inversion, Geophysical Journal International, 194(2), 1250-1280.

Kamei, R., Pratt, R. G., \& Tsuji, T., 2014. Misfit functionals in laplace-fourier domain waveform inversion, with application to wide-angle ocean bottom seismograph data, Geophysical Prospecting, 62(5), 1054-1074.

Lee, K. H. \& Kim, H. J., 2003. Source-independent full-waveform inversion of seismic data, Geophysics, 68(6), 2010-2015.

Leeuwen, T. V. \& Herrmann, F., 2013. Mitigating local minima in full-waveform inversion by expanding the search space, Geophysical Journal International, 195, 661-667.

Leeuwen, T. V. \& Herrmann, F., 2015. A penalty method for pde-constrained optimization in inverse problems, Inverse Problems, 32, 015007.

Ma, Y. \& Hale, D., 2013. Wave-equation reflection traveltime inversion with dynamic warping and full-waveform inversion, Geophysics, 78(6), R223-R233.

Pratt, R. G., 1999. Seismic waveform inversion in the frequency domain, part 1: Theory and verification in a physical scale model, Geophysics, 64(3), 888-901.

Pratt, R. G., Shin, C., \& Hick, G., 1998. Gauss-Newton and full Newton methods in frequency-space seismic waveform inversion, Geophysical Journal International, 133(2), 341-362.

R.E.Plessix, 2006. A review of the adjoint-state method for computing the gradient of a functional with geophysical applications, Geophysical Journal International, 167, 495-503.

Shin, C. \& Cha, Y. H., 2008. Waveform inversion in the laplace domain, Geophysical Journal International, 173(3), 922-931.

Skopintseva, L., Maaø, F., \& Pedersen, Ø., 2016. Importance of the source estimation in fwi-sensitivity and examples, in $78 t h E A G E$ Conference and Exhibition 2016.

Song, C. \& Alkhalifah, T., 2020a. An efficient wavefield inversion for transversely isotropic media with a vertical axis of symmetry, Geophysics, 85(3), 1-51.

Song, C. \& Alkhalifah, T. A., 2020b. Efficient wavefield inversion with outer iterations and total variation constraint, IEEE Transactions on Geoscience and Remote Sensing, pp. 1-11.

Song, C., Alkhalifah, T., Wang, G., \& Yang, Q., 2019a. An efficient wavefield inversion for isotropic elastic media, in SEG Technical Program Expanded Abstracts 2019, pp. 1575-1579, Society of Exploration Geophysicists.

Song, C., Wu, Z., \& Alkhalifah, T., 2019b. Passive seismic event estimation using multiscattering waveform inversion, Geophysics, 84(3), KS59-KS69.

Soubaras, R. \& Dowle, R., 2010. Variable-depth streamer-a broadband marine solution, first break, 28(12).

Tarantola, A., 1984. Inversion of seismic reflection data in the acoustic approximation, Geophysics, 49, 1259-1266.

Virieux, J. \& Operto, S., 2009. An overview of full-waveform inversion in exploration geophysics, Geophysics, 74(6), WCC1-WCC26.

Wang, H. \& Alkhalifah, T., 2018. Microseismic imaging using a source function independent full waveform inversion method, Geophysical Journal International, 214(1), 46-57.

Warner, M. \& Guasch, L., 2016. Adaptive waveform inversion: Theory, Geophysics, 81(6), R429-R445.

Wu, R.-S., Luo, J., \& Wu, B., 2014. Seismic envelope inversion and modulation signal model, Geophysics, 79(3), WA13-WA24.

$\mathrm{Xu}, \mathrm{K}$., Greenhalgh, S. A., \& Wang, M., 2006. Comparison of source-independent methods of elastic waveform inversion, Geophysics, 71(6), R91-R100.

Zhou, B. \& Greenhalgh, S. A., 2003. Crosshole seismic inversion with normalized full-waveform amplitude data, Geophysics, 68(4), 1320-1330. 